\title{
LLNL I/O Testbed Analysis of IBM M80 (P660 6MI)
}

\author{
K. Fitzgerald, J. Daveler, T. Heer, M. Gleicher
}

\section{February 1, 2002}

\section{U.S. Department of Energy}

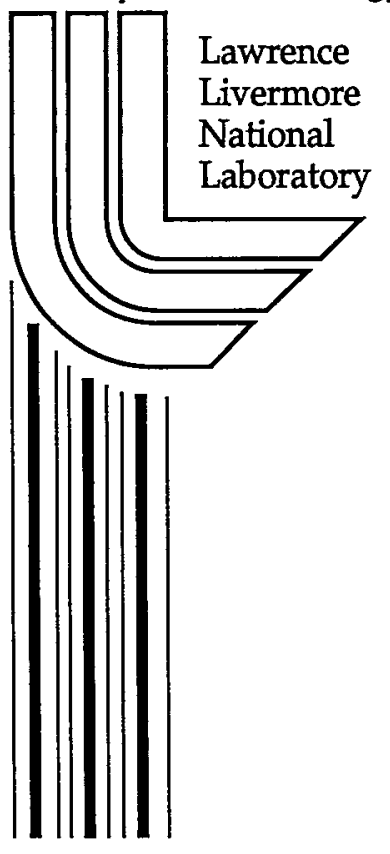




\section{DISCLAIMER}

This document was prepared as an account of work sponsored by an agency of the United States Government. Neither the United States Government nor the University of California nor any of their employees, makes any warranty, express or implied, or assumes any legal liability or responsibility for the accuracy, completeness, or usefulness of any information, apparatus, product, or process disclosed, or represents that its use would not infringe privately owned rights. Reference herein to any specific commercial product, process, or service by trade name, trademark, manufacturer, or otherwise, does not necessarily constitute or imply its endorsement, recommendation, or favoring by the United States Government or the University of California. The views and opinions of authors expressed herein do not necessarily state or reflect those of the United States Government or the University of California, and shall not be used for advertising or product endorsement purposes.

This work was performed under the auspices of the U. S. Department of Energy by the University of California, Lawrence Livermore National Laboratory under Contract No. W-7405-Eng-48.

This report has been reproduced directly from the best available copy.

Available electronically at http://www.doe.gov/bridge

Available for a processing fee to U.S. Department of Energy and its contractors in paper from

U.S. Department of Energy

Office of Scientific and Technical Information P.O. Box 62

Oak Ridge, TN 37831-0062

Telephone: (865) 576-8401

Facsimile: (865) 576-5728

E-mail: reports@adonis.osti.gov

Available for the sale to the public from

U.S. Department of Commerce

National Technical Information Service

5285 Port Royal Road

Springfield, VA 22161

Telephone: (800) 553-6847

Facsimile: (703) 605-6900

E-mail: orders@ntis.fedworld.gov

Online ordering: http://www.ntis.gov/ordering.htm

OR

Lawrence Livermore National Laboratory

Technical Information Department's Digital Library

http://www.llnl.gov/tid/Library.html 
LLNL I/O Testbed Analysis of IBM M80 (P660 6M1)

Keith Fitzgerald Jim Daveler Todd Heer Mike Gleicher

February 2002 


\section{Index}

Background: ............................................................... 3

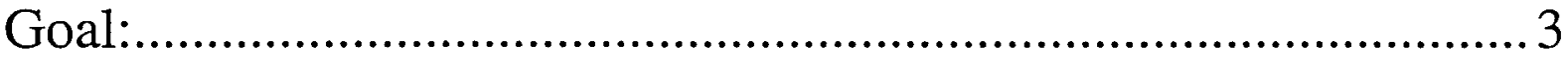

Target configuration: ........................................................... 3

Test Plan including Test results: ........................................ 8

Diagnostic simulation of HPSS behavior: (Keith Fitzgerald) ........................................ 8

HPSS Based Testing (Jim Daveler) ……………………............................................... 32

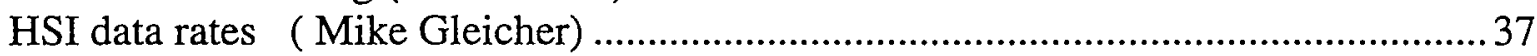

SP Attached M80 Analysis (Todd Heer) ................................................................ 42

Conclusions.................................................................... 44

Appendix A: Network options ....................................... 46

Appendix B: Outstanding Issues......................................... 48

Appendix C: Individual Card performance (reference) ............. 49 


\section{Background:}

The HPSS hierarchical storage system currently uses "mover" nodes to interface client processes to the hardware devices. As archival bandwidth requirements increase, the number of mover nodes required to provide the necessary bandwidth has become a significant factor both in terms of capital expense and administrative overhead. The LLNL data storage group would therefore like to select the most powerful yet cost effective IBM architecture currently available for use as future HPSS movers nodes. The LLNL storage group currently uses IBM "winterhawk2" SP nodes as HPSS mover nodes. Several IBM architectures including "nighthawk" SP nodes and several "P" series IBM architectures were considered and the "M80" system appeared to be the most promising based upon its high memory bandwidth and capability to attach multiple "RIO" drawers offering 64 bit $66 \mathrm{MHz}$ PCI buses.

\section{Goal:}

The goal of this testbed analysis is to determine the usefulness of the IBM M80 as an HPSS mover and to identify I/O bottlenecks in this architecture.

\section{Target configuration:}

The IBM 7026-M80 being tested is really an obsolete machine. It was replaced by the pSeries 660 Model $6 \mathrm{M} 1$ on $9 / 4 / 01$. However the hardware differences between the M80 and the P660-6M1 are all located in the processor used - NOT in the memory or I/O areas. In fact, current IBM M80 customers can upgrade their systems to the new model architecture by replacing their current processor cards much the way we upgraded from nighthawk1 to nighthawk 2 systems. Therefore since our goal is to saturate the $\mathrm{I} / \mathrm{O}$ and/or memory capability of the machine and the new $6 \mathrm{M} 1$ systems are not available for borrows, we believe our $\mathrm{I} / \mathrm{O}$ test results will be valid and we can extrapolate results in the processor area. The following diagrams were found in the "IBM eserver pseries 660 model 6M1 Technical Overview and Introduction. Written by Stephen Lutz and Shyam Manohar September 4,2001. 


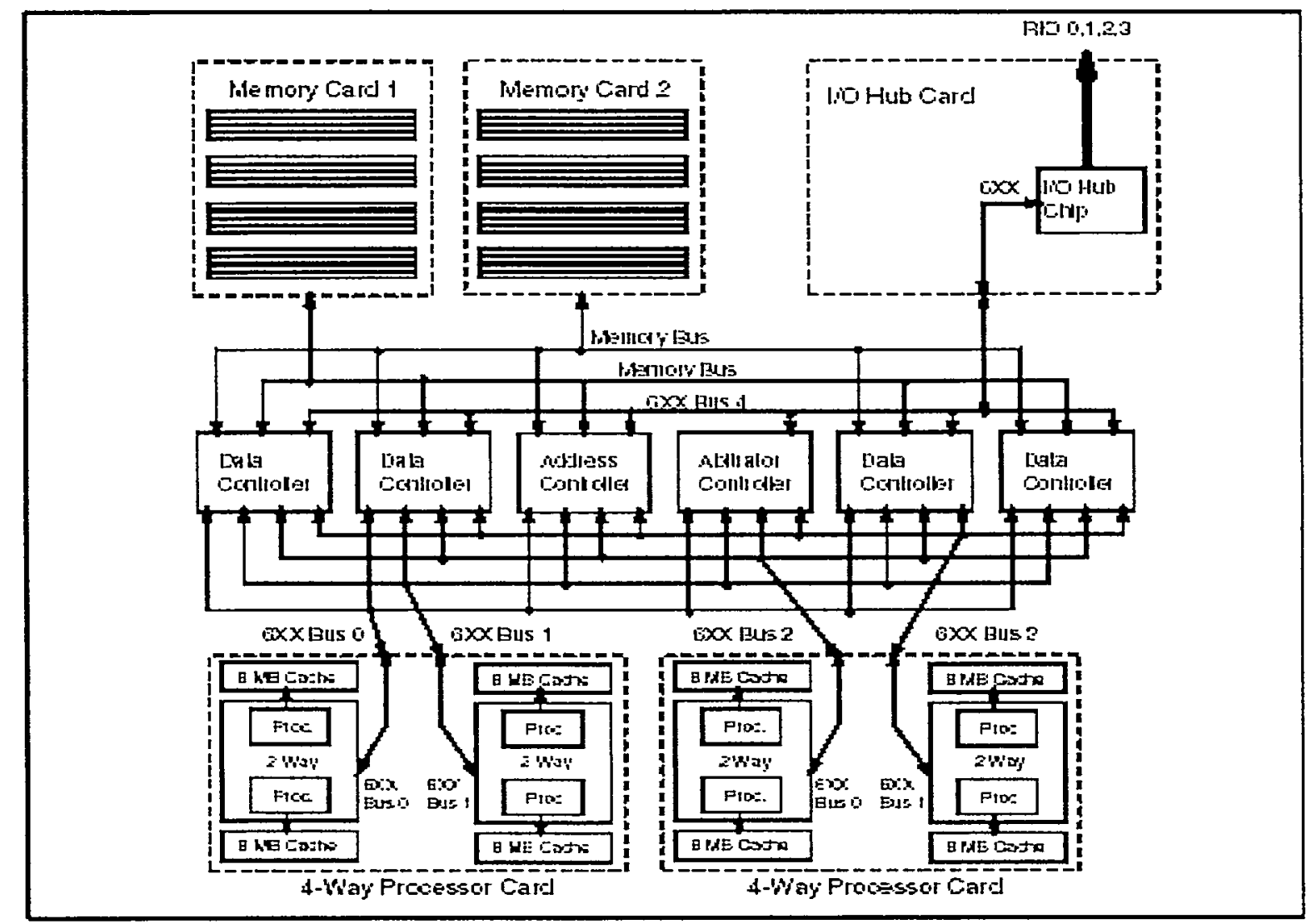

Figure 2. BS:6000 Madel 6ut System Schematic within the CEC.

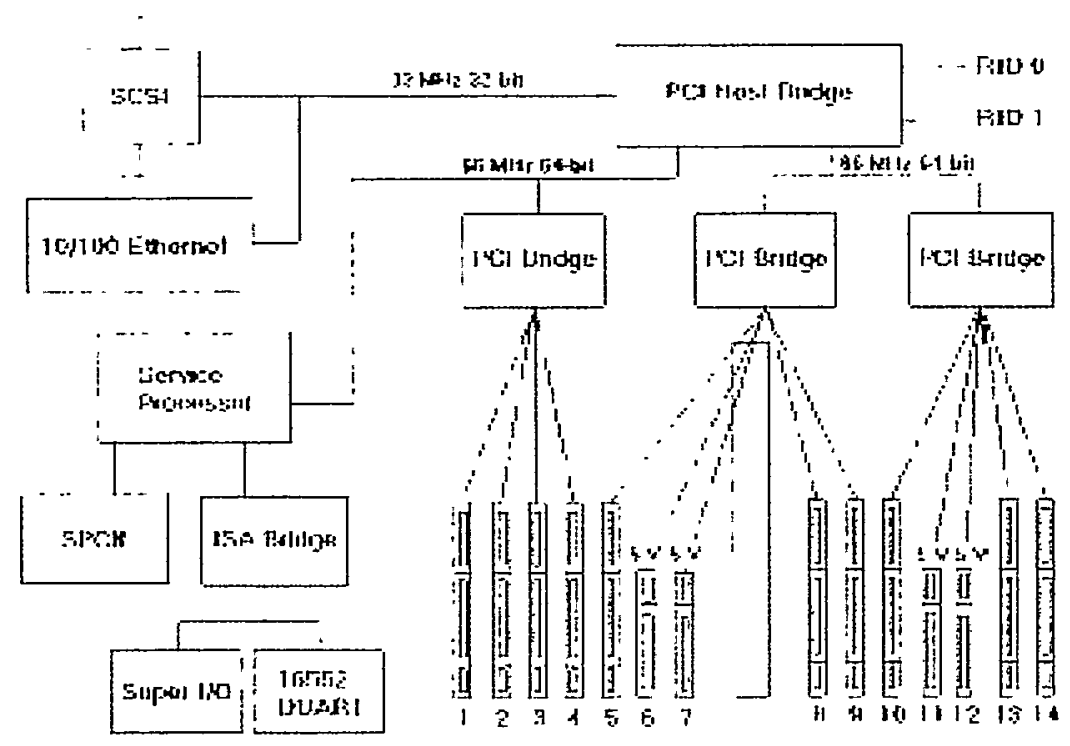

Figure 5. Siot Layout of the ro Dinver 


\section{Bus Bandwidth}

The following are the theoretical maximum bandwidths, as applicable for an 8-way $750 \mathrm{MHz}$ SMP configurations:

- Bandwidth of the bus between each memory riser card to memory controller: 4.8 GB/s

- Bandwidth of the PowerPC $6 x x$ bus used to interface each pair of processors: $2.4 \mathrm{~GB} / \mathrm{s}$

- Bandwidth of the PowerPC 6xx bus used to interface the $1 / 0$ hub: $2.4 \mathrm{~GB} / \mathrm{s}$

- Aggregate memory bandwidth: $9.6 \mathrm{~GB} / \mathrm{s}$

- Aggregate processor bandwidth: $9.6 \mathrm{~GB} / \mathrm{s}$

- Four drawer I/O bandwidth: $4 \mathrm{~GB} / \mathrm{s}(4 \times 500 \mathrm{MB} / \mathrm{s}$ bi-directional)

Analysis of the system architecture leads us to believe that the worst restriction in the M80 is the $2 \mathrm{~GB} / \mathrm{s} 6 \mathrm{XX}$ bus that connects the $\mathrm{I} / \mathrm{O}$ hub to the memory controllers (16 bytes@125Mhz $=2 \mathrm{~GB} / \mathrm{s}$ ). If we assume the same bus utilization that we achieved on the winterhawk nodes $(300 \mathrm{MB} / \mathrm{s}$ of a possible $480 \mathrm{MB} / \mathrm{s}=63 \%)$ that means we can expect $1,260 \mathrm{MB} / \mathrm{s}$ on the $\mathrm{M} 80$ if this is indeed the choke point. $1260 \mathrm{MB} / \mathrm{s}$ translate to a quantity of roughly $12 \mathrm{I} / \mathrm{O}$ interfaces each running at $100 \mathrm{MB} / \mathrm{s}$. Each RIO loop can run at $1 \mathrm{~GB} / \mathrm{s}$ in each direction $(500 \mathrm{MB} / \mathrm{s} * 2$ connections) - therefore two RIO drawers should give us $2 \mathrm{~GB} / \mathrm{s}$ (theoretical) in each direction - more than enough to saturate the $6 \mathrm{XX}$ bus. Within each RIO drawer you have two 64 bit $66 \mathrm{Mhz}$ PCI buses. Each PCI bus should be capable of running at $528 \mathrm{MB} / \mathrm{s}$ and should actually be able to achieve around $60 \%$ of the theoretical rate or $316 \mathrm{MB} / \mathrm{s}$. If we install three $100 \mathrm{MB} / \mathrm{s} \mathrm{I} / \mathrm{O}$ cards in each PCI bus and use two RIO drawers, we should have a configuration which can saturate the $6 \mathrm{XX}$ I/O bus leading from the I/O Hub card to memory while transferring data at full speed in either direction (12cards * 100MB/s each). The proposed target configuration follows. Due to the full duplex nature of the cards, the machine is actually oversubscribed by a factor of 2 . Note that we installed like cards in each PCI bus so a device with a high interrupt priority should not be able to starve the other cards on the PCI bus. 


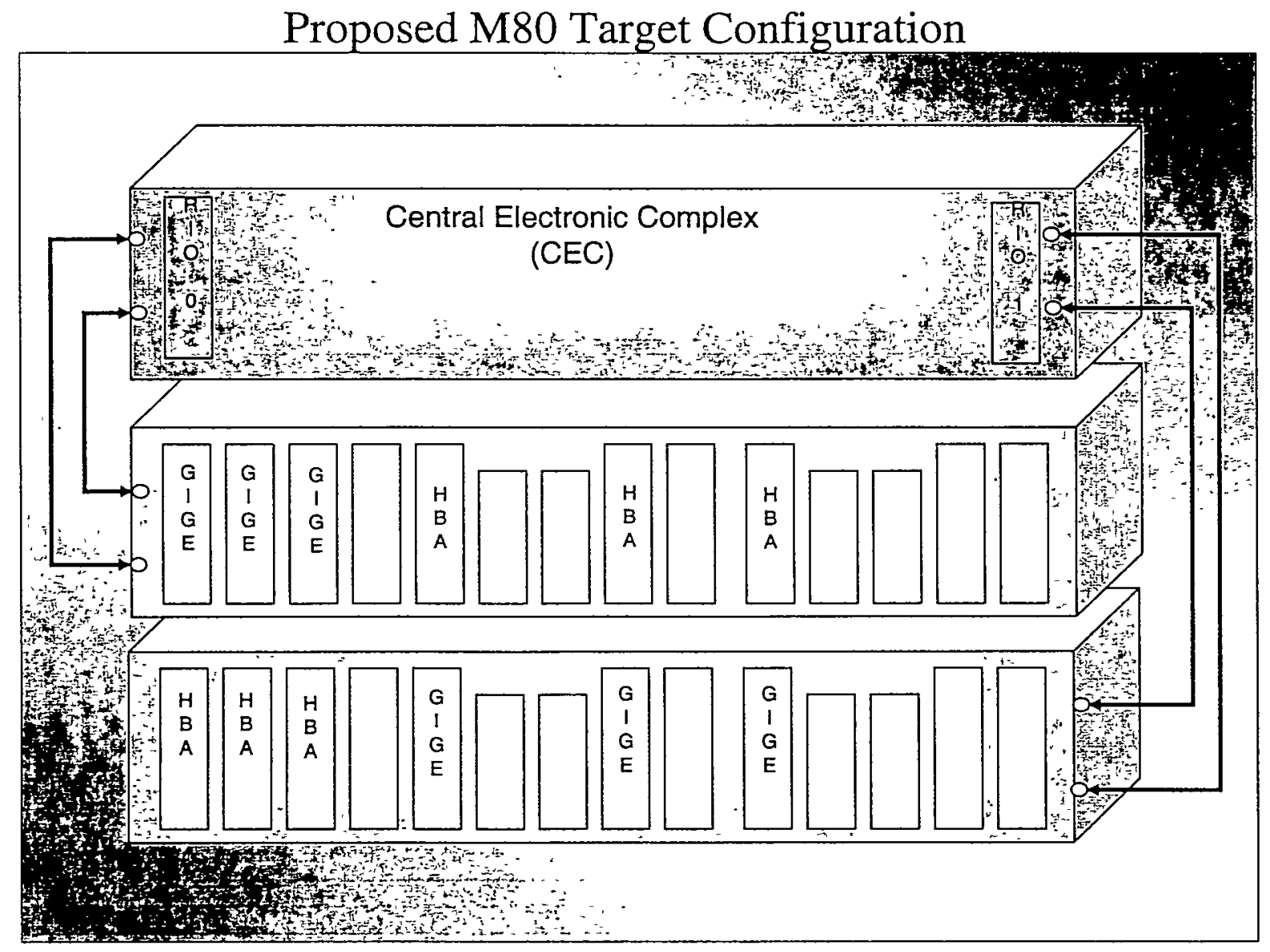

A series of tests will be run which will hopefully verify the proposed configuration. The goal of the testing will be to find the point at which the I/O capability of the hardware has been exhausted. However before beginning the L/O stress tests we will attempt to verify that each I/O device, PCI bus, and RIO drawer performs as expected when tested individually. Once baseline performance has been verified, we will run a series of tests designed to incrementally increase the $\mathrm{V} / \mathrm{O}$ load on the test configuration until the throughput peaks. Past experience has shown us that $\mathrm{I} / \mathrm{O}$ throughput dramatically degrades when the system saturates. Once we have determined the optimal I/O load for the test configuration we will attempt to determine the number of processors and memory required to support the I/O load. We will then configure the test configuration as an HPSS mover in order to determine how closely our test results match actual system behavior. As a result of the testing will be an optimized mover configuration, which will deliver a known I/O performance. Since our test configuration will hopefully support (at least) six gige interfaces we will have to configure two additional jumbo gige subnets in the I/O testbed.

IBM Hardware: 
IBM M80 with 8 processors, 32 GB memory and 3 additional RIO drawers (4 total).

LLNL hardware:

6 IBM gigabit Ethernet cards

6 emulex LP8000 Host Bus Adapters

Brocade 4400 16-port fibre switch

2 TB Data Direct Networks (DDN) fibre RAID disk. (4 tiers capable of running at $96 \mathrm{MB} / \mathrm{s}$ on each interfaces)

1 TB MetaStor fibre RAID disk ( 2 interfaces capable of running at $80 \mathrm{MB} / \mathrm{s} \mathrm{each}$ )

1 IBM nighthawk2 16 processor 8GB memory SP node with two RIO drawers (two gigabit interfaces in each)

4 IBM winterhawk 4 processors 2 GB memory SP nodes each with one jumbo gigabit Ethernet interface. 


\section{Test Plan including Test results:}

(see actual test log for more specific information).

\section{Diagnostic simulation of HPSS behavior: (Keith Fitzgerald)}

1. Install and update operating system patch level.
AIX version 4.3.3 was installed and updated to level 50 .
$</$ u/keith>lslpp - 1 | grep bos
bos.rte.bosinst 4.3 .3 .50
COMMITTED Base os Install

2. Test Memory bandwidth

Historically, we've found a high correlation between memory bandwidth and performance as an HPSS mover. The "Memtest" program exercises memory by allocating two buffers and copying data between them. You increase the number of concurrent processes until the machine's memory bandwidth is saturated. We have data on silver nodes, winterhawk nodes, and nighthawk nodes which can be compared to the M80 performance. 
Tested memory using "memtest" 10MB buffer 100 copies

\begin{tabular}{|c|c|c|c|c|c|c|c|c|c|}
\hline Processors & 1 & 2 & 3 & 4 & 5 & 6 & 7 & 8 & 16 \\
\hline $\begin{array}{c}\text { Bandwidth } \\
(\mathrm{MB} / \mathrm{s})\end{array}$ & 186 & 369 & 548 & 689 & 840 & 977 & 1098 & 1202 & 1216 \\
\hline
\end{tabular}

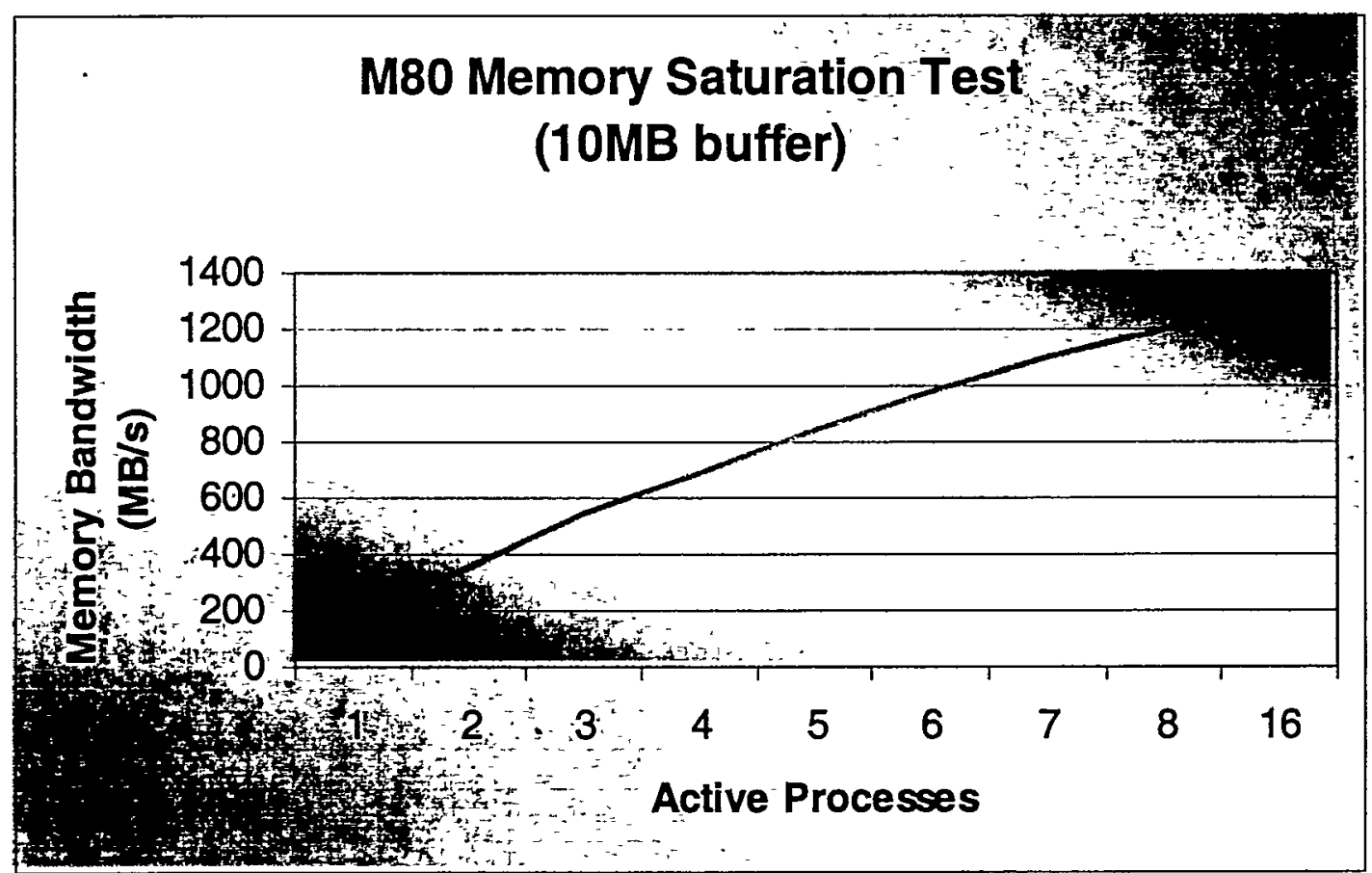

Note that there's enough memory bandwidth for all processors (meaning that the data rate increases as each process is added, asymptotically approaching the memory saturation value). Aggregate bandwidth increases as each physical processor becomes active.

The following graph compares M80 memory bandwidth to other architectures we have tested. A 16 processor Nighthawk2, a 4 processor Winterhawk2, and a 4 processor Silver Node. 


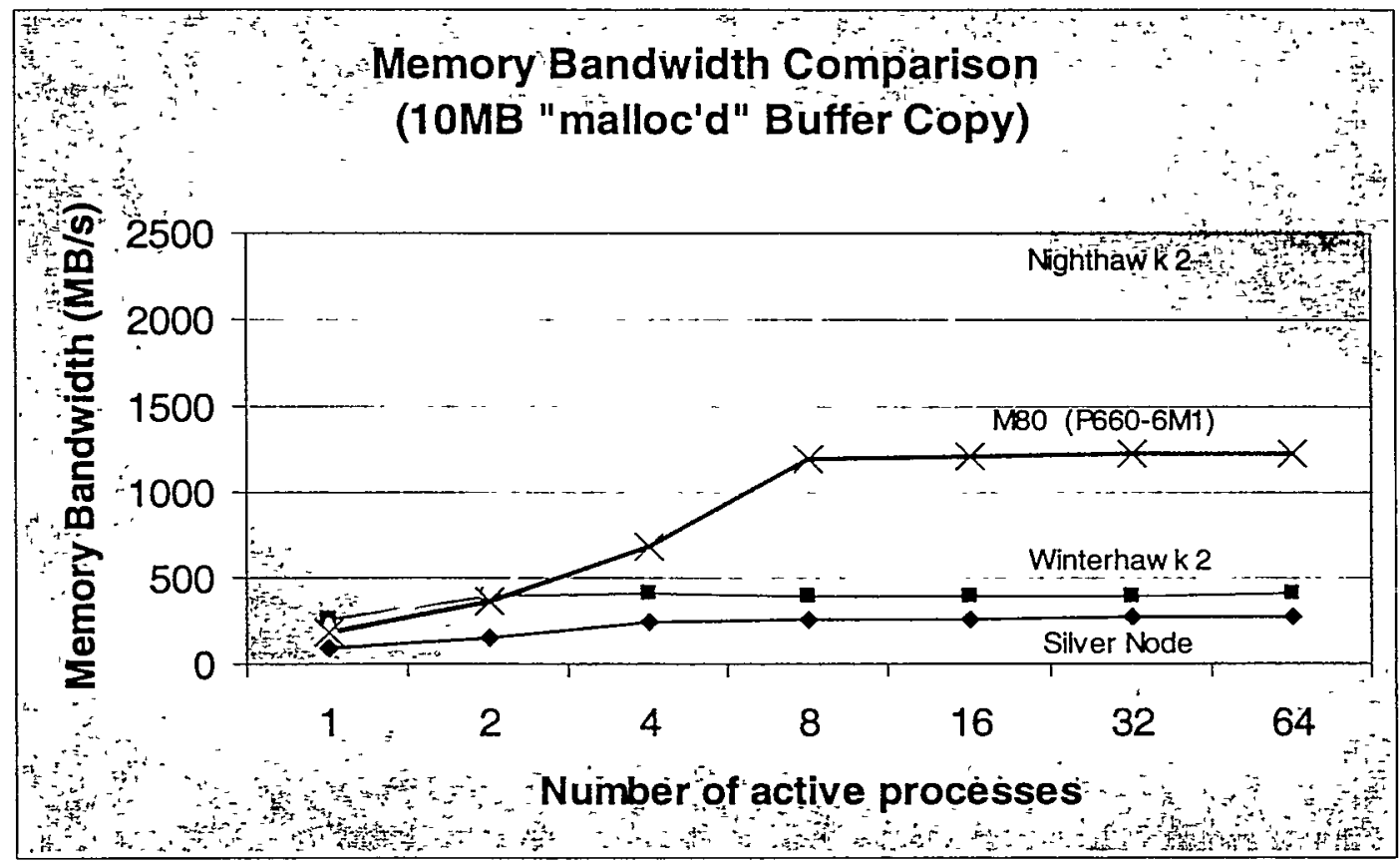


3. Installed four IBM gigabit Ethernet interfaces in the first PCI bus (slots $1,2,3,4$ ) of the RIO drawer daisy chained to the primary RIO drawer.

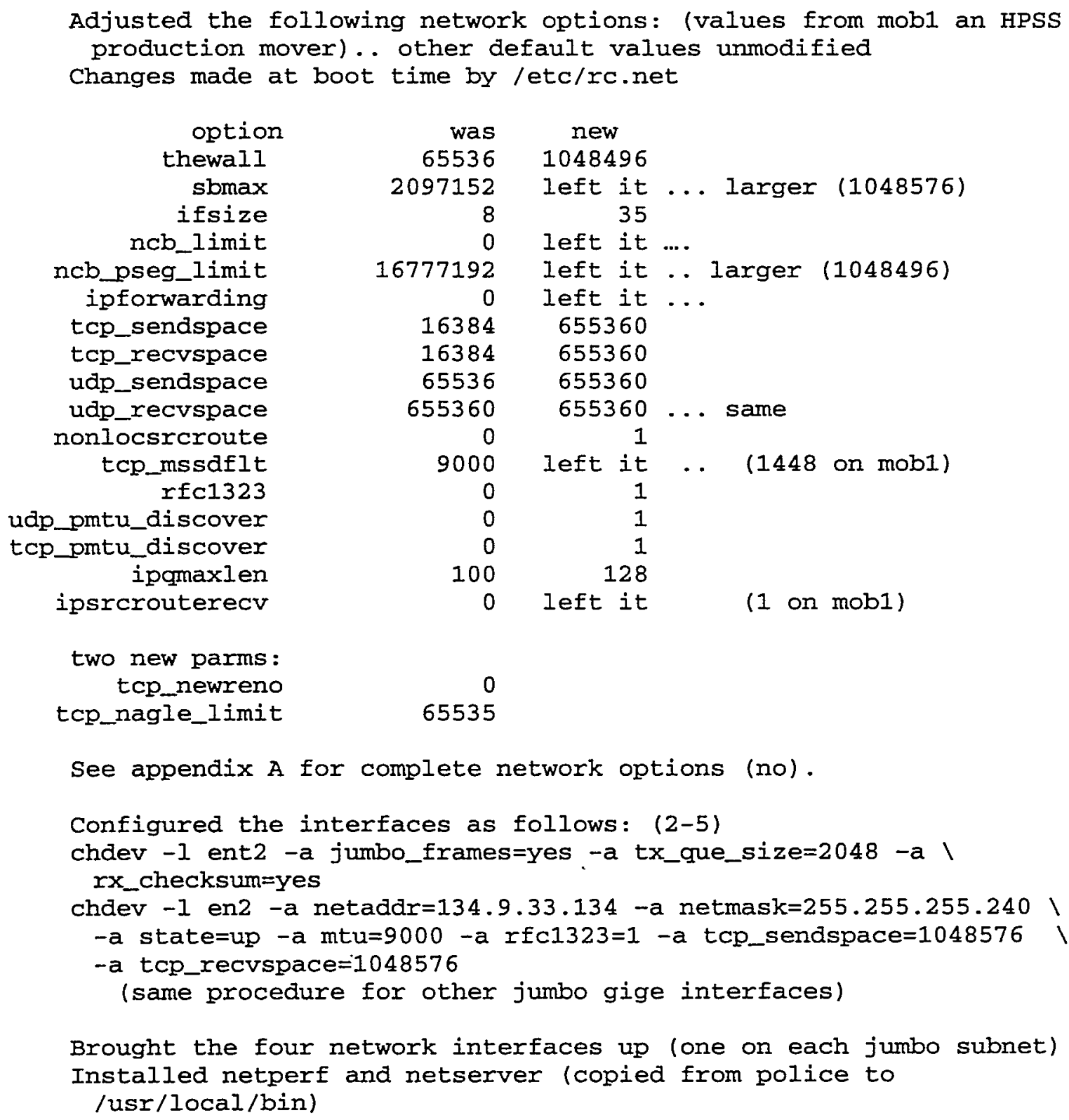

The following series of tests will be performed using diagnostic routines (netperf for gige cards and a simple "home grown" memory based disk test which selectively reads or writes large blocks of disk data).

LLNL runs four gigabit Ethernet subnets in our production environment. The I/O testbed is configured to mirror our production environment as much as possible. In the following diagram, the M80 (inhale) has four gigabit interfaces, one configured on each of the four jumbo subnets. Our test environment also included a nighthawk2 node (wood1) which also supports four jumbo gigabit Ethernet interfaces. The four winterhawk2 nodes (mob1-4) each have a single jumbo gigabit Ethernet interface and are 
configured one on each of the four jumbo Ethernet subnets. On inhale, en2-5 were the jumbo subnets.

\begin{tabular}{|c|c|c|c|}
\multicolumn{1}{c}{ Jumbo subnet } & M80 interface & Nighthawk & Winterhawk \\
\hline 1 & inhale-en2 & wood1-en2 & mob1-en2 \\
\hline 2 & inhale-en3 & wood1-en3 & mob2-en2 \\
\hline 3 & inhale-en4 & wood1-en4 & mob3-en2 \\
\hline 4 & inhale-en5 & wood1-en5 & mob4-en2 \\
\hline
\end{tabular}


4. Verify individual data rates of each M80 gige adapter. We used the first PCI bus in the RIO drawer daisy chained to the primary RIO. This test used the winterhawk nodes as data source or sink because they are completely independent.

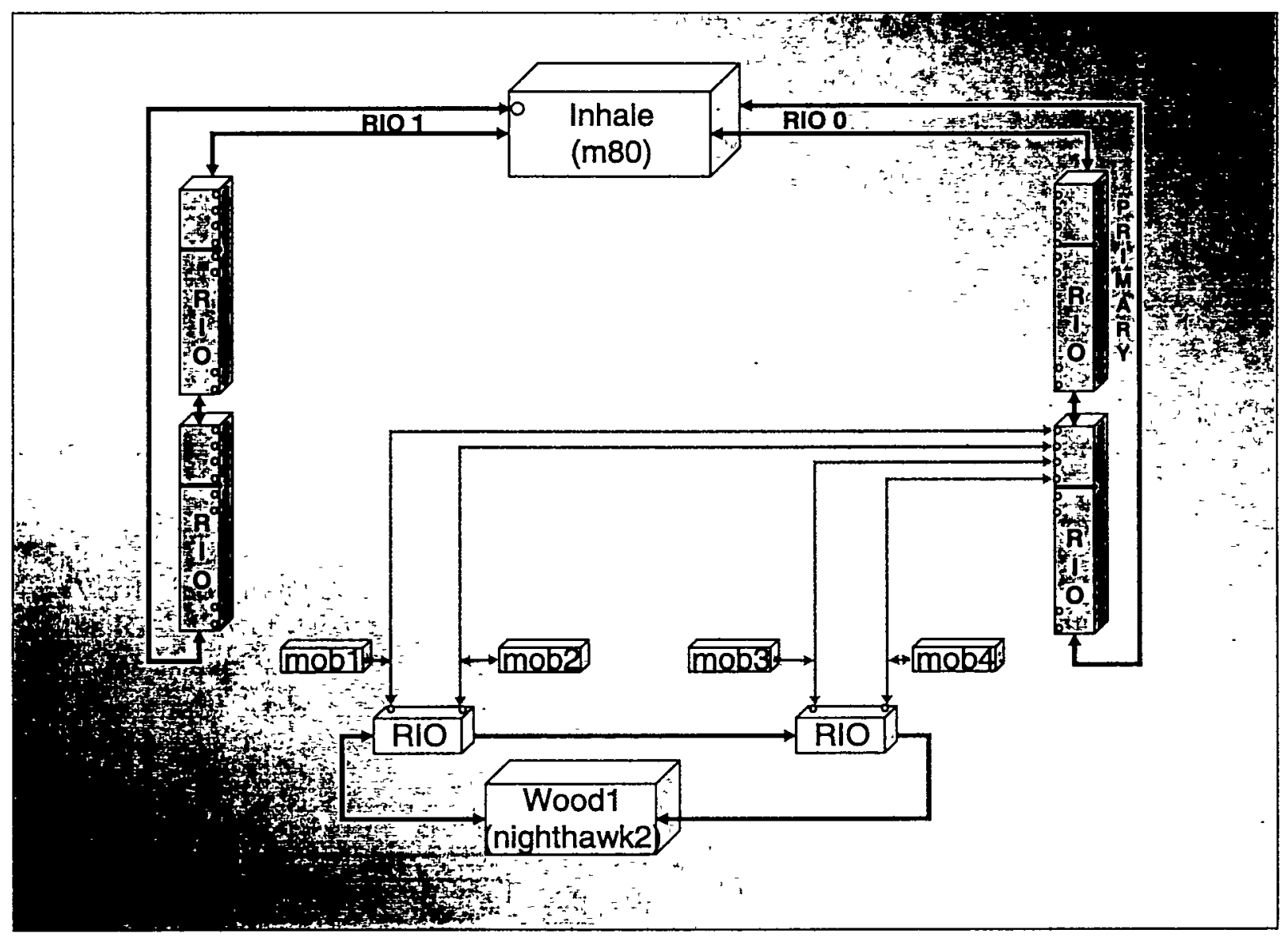

\begin{tabular}{|c|c|c|c|}
\multicolumn{1}{c}{ Source } & Destination & $\begin{array}{c}\text { Data rate } \\
\text { (MB/s) }\end{array}$ & $\begin{array}{c}\text { CPU Utilization } \\
\text { (M80 perspective) }\end{array}$ \\
\hline inhale-en2 & mob1-en2 & 88 & $7 \%$ \\
\hline inhale-en3 & mob2-en2 & 88 & $7 \%$ \\
\hline inhale-en4 & mob3-en2 & 88 & $7 \%$ \\
\hline inhale-en5 & mob4-en2 & 85 & $7 \%$ \\
\hline mob1-en2 & inhale-en2 & 100 & $9 \%$ \\
\hline mob2-en2 & inhale-en3 & 100 & $9 \%$ \\
\hline mob3-en2 & inhale-en4 & 100 & $9 \%$ \\
\hline mob4-en2 & inhale-en5 & 100 & $9 \%$ \\
\hline
\end{tabular}

Note that at this point $I$ found that the jumbo gige cards in the four winterhawk 2 nodes (mob1-mob4) were located in the 32 bit $33 \mathrm{MHz}$ PCI bus. This impacted the peak and full duplex performance of the gige cards installed in the M80. The winterhawks were 
reconfigured and the jumbo interface moved to one of the 64 bit $33 \mathrm{MHz}$ PCI buses later in the testing.

5. Saturate PCI bus by activating gige cards one at a time and re-testing performance until full device bandwidth can not be achieved

The following graphs show that using IBM gigabit Ethernet adapters a $64 \mathrm{bit} 66 \mathrm{MHz}$ $\mathrm{PCI}$ bus saturates at around three concurrent transfers. Maintaining three transfers requires around $35 \% \mathrm{cpu}$ utilization (35\% of $8 \mathrm{cpu}$ 's) for a read or $25 \%$ when writing.

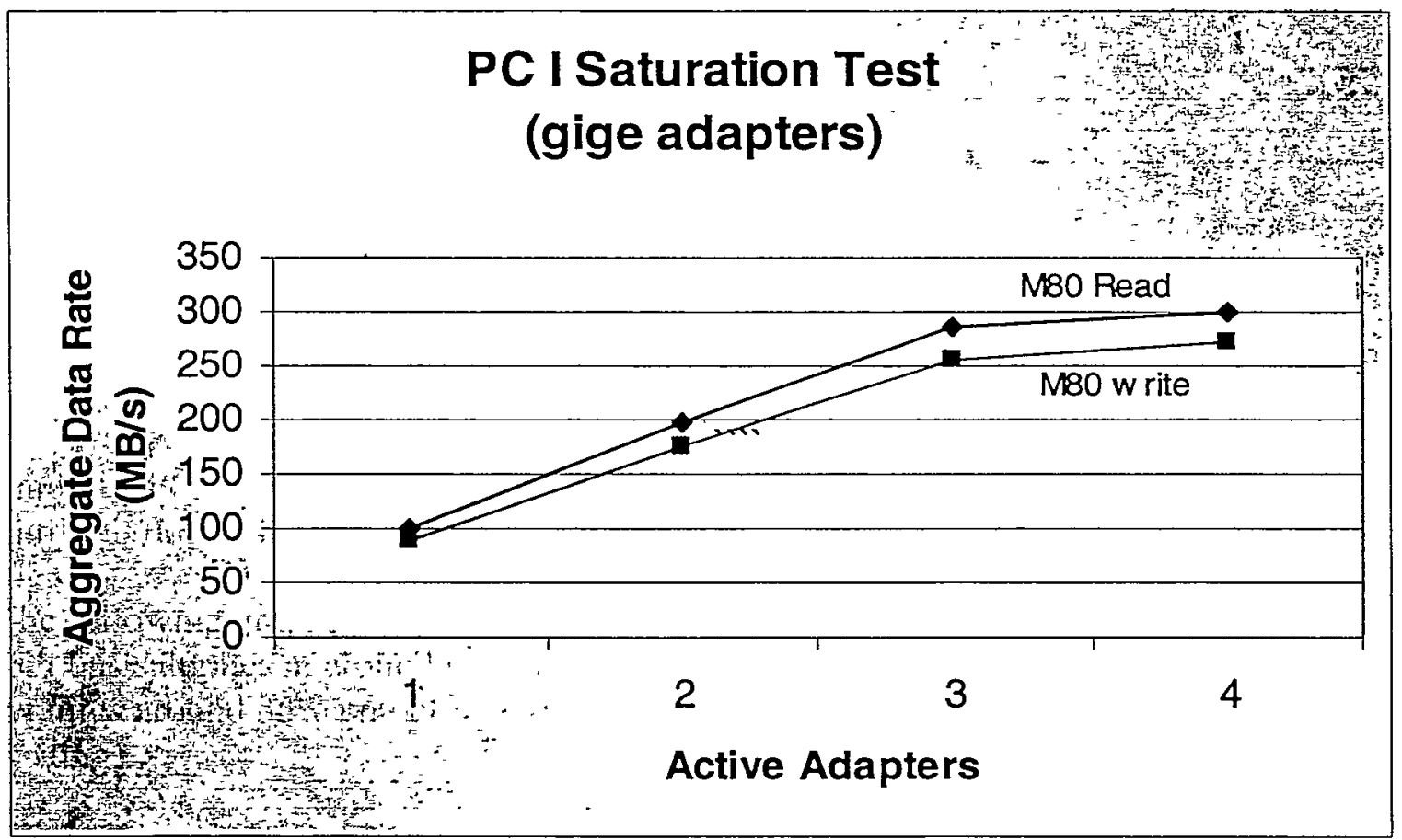




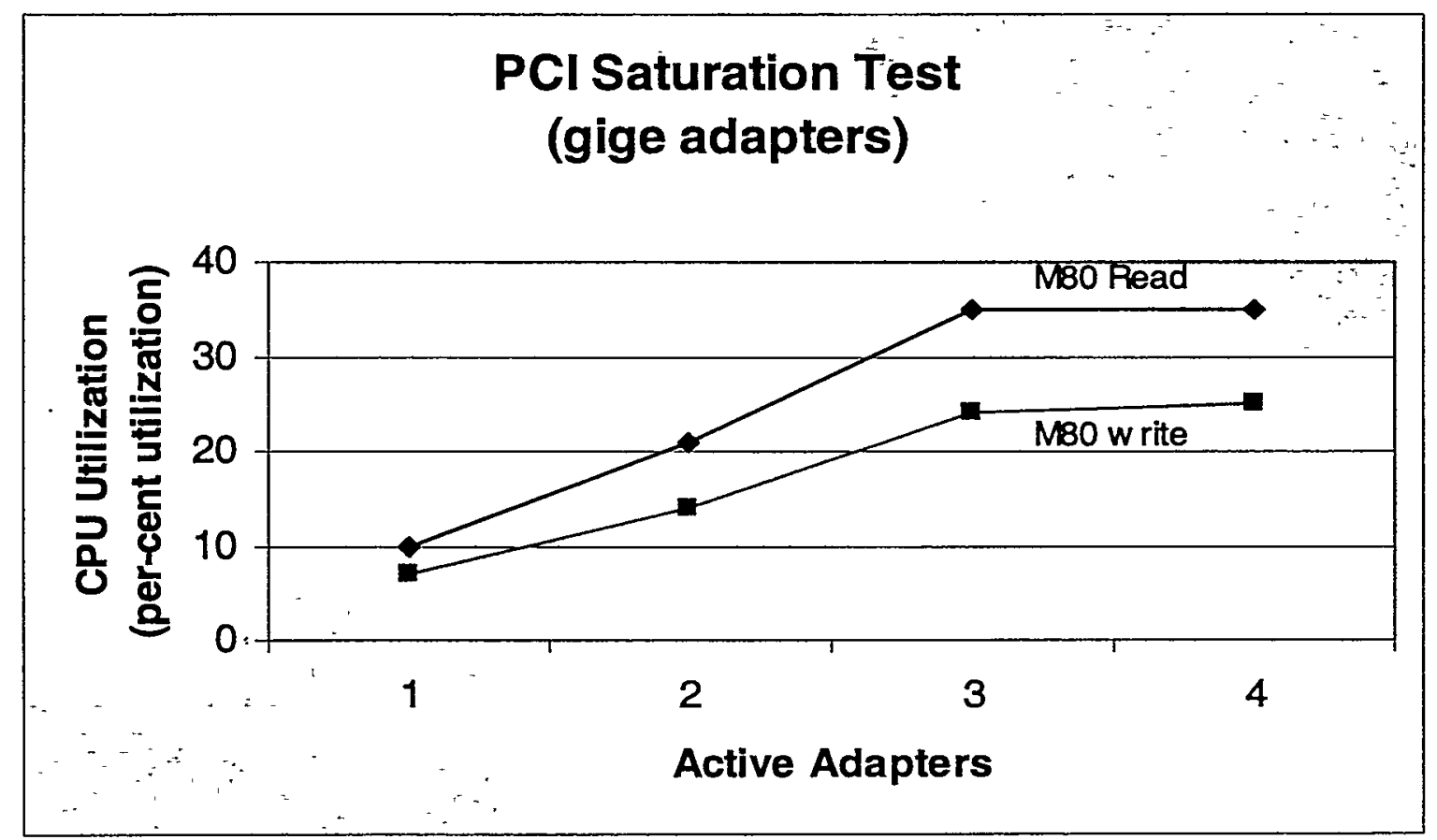


6. Remove the four gige adapters and replace them with four emulex LP8000 Host Bus Adapters (HBA's). Verify the performance of each emulex HBA and the associated disk device. (same goal as step 4 above).

Installed emulex version 4.1.0.5 fibre channel driver. Upgraded emulex HBA microcode to d382al.awc (kernel revision LP8K $2.03 \times 15$ ) (initially got bad rates on 3 down level adapters!)

changed the following default parameters for fcosdisk: attribute=mode_data default $d="$ " (was a hex \#) attribute=queue_depth default $=64$ (was 8)

changed the following default parameter in lp8000 attribute=lun_queue_depth default $=64$ (was 30)

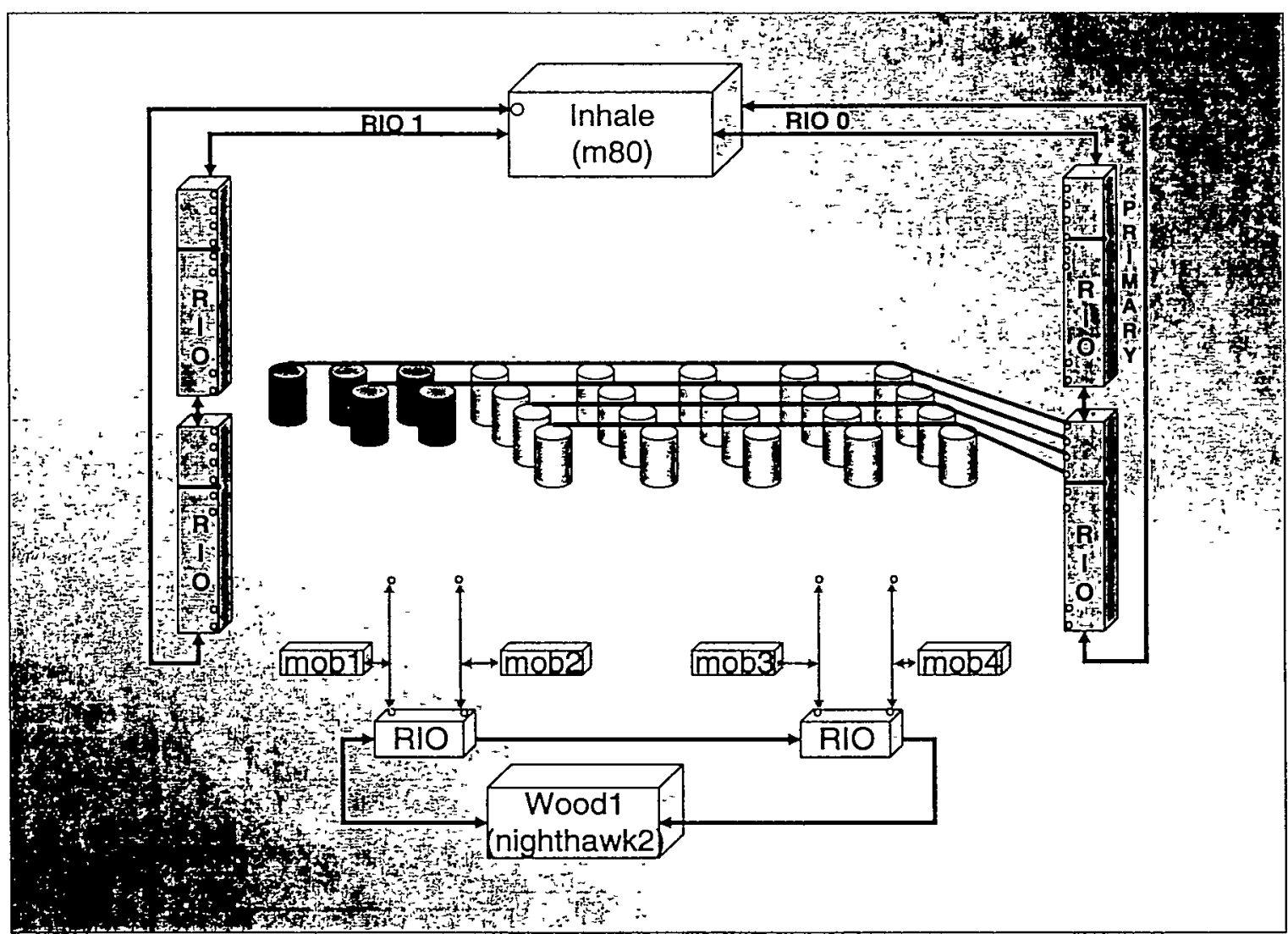

I utilized the M80 "hot swap" capability to remove the gige adapters and install the HBA's. It worked great and the M80 RIO drawer design is a big improvement over the nighthawk RIO. In the actual test configuration, the M80's HBA's were connected to a 16 port Brocade 2801 fibre switch. All disk devices were also attached to the switch. This allowed me to verify disk behavior from the winterhawk2 nodes without any recabling or reconfigurations. Although multiple logical units (LUN's) were configured on each physical RAID device, care was taken 
during testing to assure that no more than one data stream was active to each physical device. Our network attached disk test environment includes four "tiers" (physical RAID devices) of Data Direct Networks (DDN) disk, each with a dedicated fibre interface. The DDN disks are capable of reading and writing at $96 \mathrm{MB} / \mathrm{s}$ based upon tests using our IBM winterhawk2 nodes and emulex LP8000 HBA's via the Brocade switch. We also have a "metastor" fibre disk device with two fibre interfaces. The "metastor" disk is capable of reading and writing at about $80 \mathrm{MB} / \mathrm{s}$, again verified using our IBM winterhawk2 nodes, using emulex LP8000 adapters and the brocade switch.

\begin{tabular}{|c|c|c|c|}
\hline & $\begin{array}{l}\text { READ } \\
(\mathrm{MB} / \mathrm{s})\end{array}$ & $\begin{array}{l}\text { WRITE } \\
(\mathrm{MB} / \mathrm{s})\end{array}$ & $\begin{array}{c}\text { READ/WRITE } \\
(\mathrm{MB} / \mathrm{s})\end{array}$ \\
\hline $\begin{array}{c}\text { Winterhawk data rates } \\
\text { (baseline) }\end{array}$ & 98 & 98 & $150-160$ \\
\hline M80 data rates & 98 & 77 & 112 \\
\hline
\end{tabular}

These tests used an $8 \mathrm{MB}$ read/write size in the test program. M80 HBA write rates are VERY low! We get 98 from our winterhawk2 nodes. We are currently working with emulex to resolve the problem. During these individual HBA tests, I also discovered that the LP8000's full duplex performance is also bad on the M80. We later installed and tested an emulex LP9000 and saw very similar performance. 
7. Saturate PCI bus by adding disk streams (unique HBA and disk device) one at a time and re-testing performance until full device bandwidth can not be achieved. (corresponds to step4 in the gige testing).
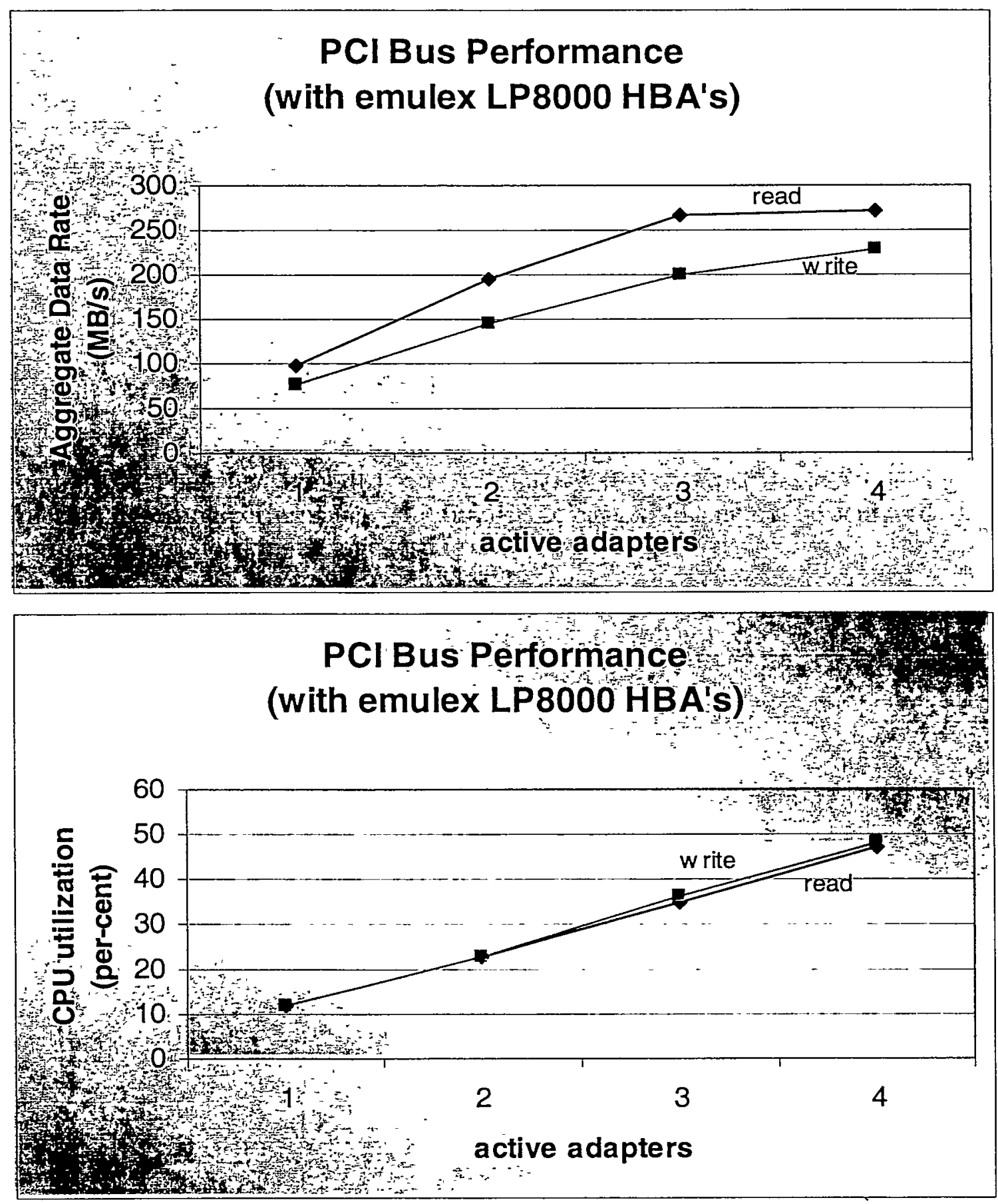
This test also showed that three adapters can operate at (or near) full speed concurrently running half-duplex. A fourth adapter saturates the PCI bus. I also attempted to saturate the PCI bus with a combination of reads and writes.

I also ran a test that wrote to all four DDN disks while concurrently reading the metastor disks. Two HBA's were reading AND writing concurrently. Two HBA's were just writing. I achieved a total of $89 \mathrm{MB} / \mathrm{s}$ on the two reads and a total of $161 \mathrm{MB} / \mathrm{s}$ on the four writes for a total of $250 \mathrm{MB} / \mathrm{s}$ and $68 \% \mathrm{CPU}$ utilization. Note that the CPU utilization (wait time) increased even tho the aggregate bandwidth did not.

\begin{tabular}{|c|r|r|r|}
\multicolumn{1}{c}{} & \multicolumn{1}{c}{$\begin{array}{c}\text { Read } \\
\text { Adapter }\end{array}$} & \multicolumn{1}{c}{$\begin{array}{c}\text { Write } \\
(\mathrm{MB} / \mathrm{s})\end{array}$} & $\begin{array}{c}\text { Total } \\
(\mathrm{MB} / \mathrm{s})\end{array}$ \\
\hline lpfc0 & 44 & 31 & 75 \\
\hline lpfc1 & 45 & 30 & 75 \\
\hline lpfc2 & - & 51 & 51 \\
\hline lpfc3 & - & 49 & 49 \\
\hline \multicolumn{3}{|r|}{}
\end{tabular}


8. Verify that both buses in an individual RIO drawer can run at full speed concurrently.

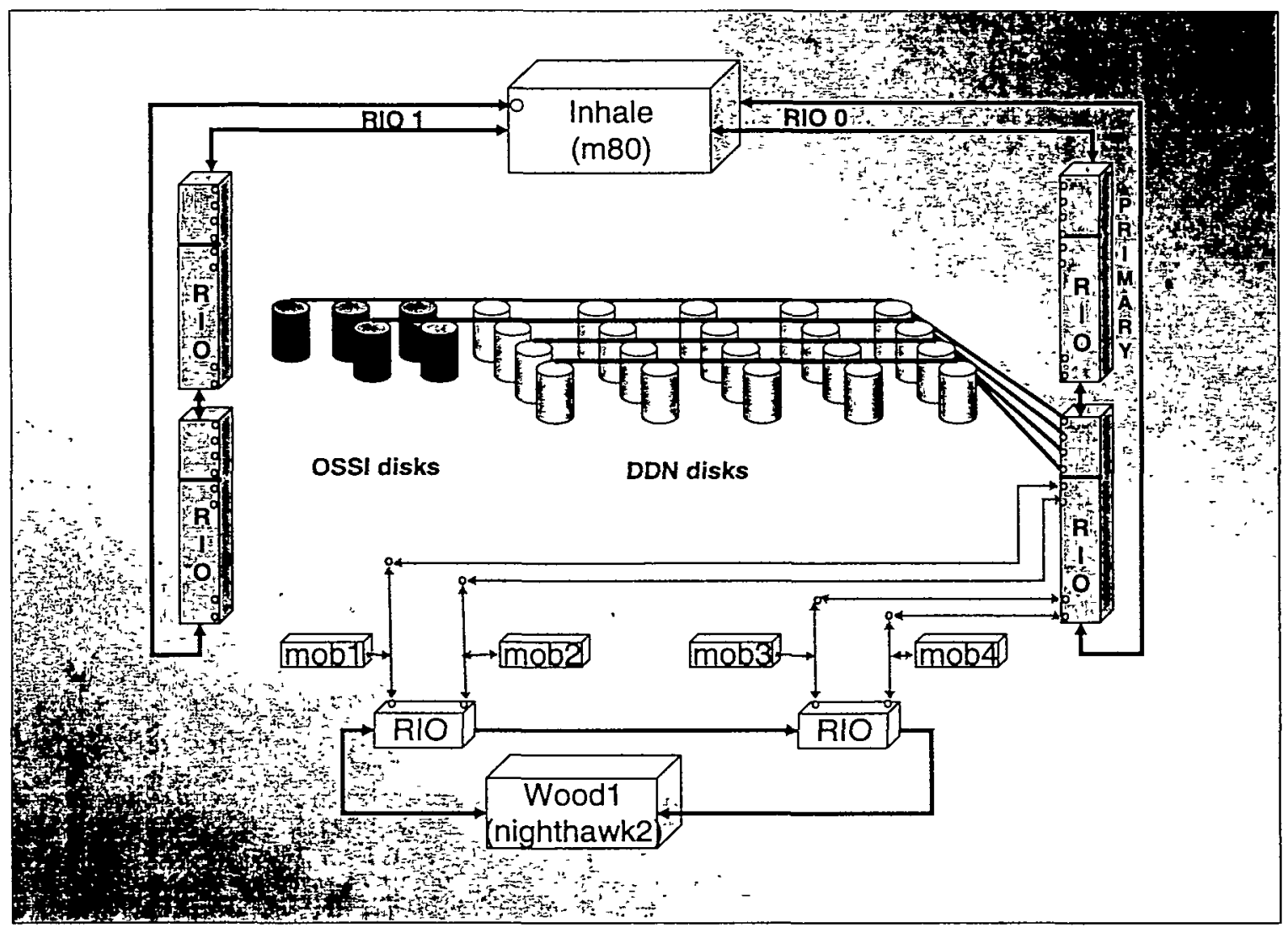

I installed the four IBM gige cards tested in step 3 in the $2^{\text {nd }} \mathrm{PCI}$ bus of the RIO drawer containing the four LP8000 HBA's. After verifying the individual performance of the gige cards in their new location, I attempted to run all four gige cards concurrently. I found that one of the cards performed at full speed and the other three cards split the remaining bandwidth. I believe that this is caused by the PCI bus repeaters. The bridge repeaters seem to distribute the bandwidth available on the primary bus evenly to the two secondary buses. I achieved around $325 \mathrm{MB} / \mathrm{s}$ total reading from all four interfaces concurrently.

Interface

3

4

active interfaces active interfaces

\begin{tabular}{|c|c|c|}
\hline en2 (61-08) & 98 & 74 \\
\hline en3 (6A-08) & 99 & 74 \\
\hline en4 (6D-08 & 99 & 74 \\
\hline en5 (71-08) & - & 102 \\
\hline
\end{tabular}

This is an interesting discovery but not the relevant to the test. It is, however the highest aggregate PCI bus data rate so far....

Theoretical PCI bus rate $=8$ bytes $/$ cycle $* 66 \mathrm{M}$ cycles $/ \mathrm{sec}=528 \mathrm{MB} / \mathrm{s}$

PCI bus saturation $=324 / 528=61 \%$ theoretical 
Now that the gige performance had been verified, I setup a test that read four DDN disks on the first bus while concurrently reading four gige adapters on the second PCI bus. Got a total of $375 \mathrm{MB} / \mathrm{s}$... with $25 \%$ CPU utilization.

RIO Saturation READ test

\begin{tabular}{|c|c|c|c|}
\multicolumn{1}{c|}{ PCI bus } & Adapter & Device & Rate \\
\hline 1 & lpfc0 & DDN-tier1 & 41 \\
\hline 1 & lpfc1 & DDN-tier2 & 42 \\
\hline 1 & lpfc2 & DDN-tier3 & 43 \\
\hline 1 & lpfc3 & DDN-tier4 & 43 \\
\hline 2 & en2 & mob1-en2 & 41 \\
\hline 2 & en3 & mob2-en2 & 41 \\
\hline 2 & en4 & mob3-en2 & 41 \\
\hline 2 & en5 & mob4-en2 & 83 \\
\hline
\end{tabular}

Then I tried the same thing with writes $325 \mathrm{MB} / \mathrm{s}$ with $17 \%$ CPU utilization.

RIO Saturation WRITE test

\begin{tabular}{|c|c|c|c|}
\hline PCI bus & Adapter & Device & Rate \\
\hline 1 & $\operatorname{lpfc0}$ & DDN-tier1 & 40 \\
\hline 1 & lpfc1 & DDN-tier2 & 38 \\
\hline 1 & $\operatorname{lpfc2}$ & DDN-tier3 & 40 \\
\hline 1 & lpfc3 & DDN-tier4 & 40 \\
\hline 2 & en 2 & mob1-en2 & 40 \\
\hline 2 & en3 & mob2-en2 & 40 \\
\hline 2 & en4 & mob3-en2 & 40 \\
\hline 2 & en5 & mob4-en2 & 47 \\
\hline
\end{tabular}

THIS IS SIGNIFICANT ...the rates achieved indicate that an individual RIO drawer can not make use of both RIO connections! The RIO daisy chain architecture seems to only add redundancy - you can't seem to make use of the bandwidth! I suspect that you will have to add the daisy-chained RIO drawer in order to make use of the second RIO connection to the CEC.

A RIO connection's theoretical data rate is $500 \mathrm{MB} / \mathrm{s}$.

RIO connection saturation seems to be $375 / 500=75 \%$ theoretical 
9. Verify that the daisy chained RIO drawer can make use of the second RIO connection while the first RIO uses the first connection.

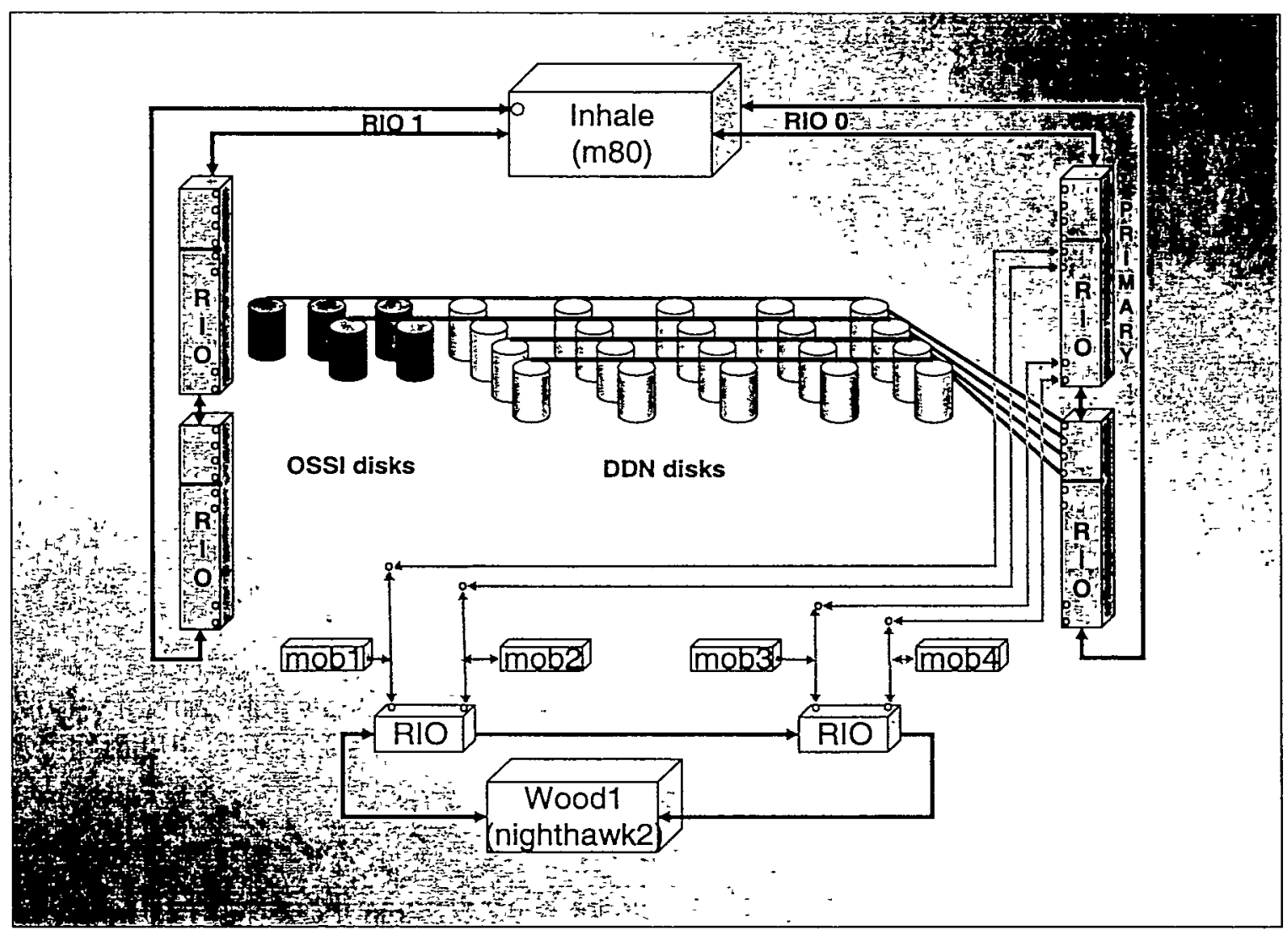

To test this theory I moved the four gige cards to the primary RIO drawer and reran the previous test sequence.

Got $554 \mathrm{MB} / \mathrm{s}$ reading 8 devices with $44 \%$ cpu utilization.

RIO Saturation READ test

\begin{tabular}{|c|c|c|c|}
\multicolumn{1}{c|}{ PCI bus } & Adapter & Device & Rate \\
\hline 1 & lpfc0 & DDN-tier1 & 51 \\
\hline 1 & lpfc1 & DDN-tier2 & 74 \\
\hline 1 & Ipfc2 & DDN-tier3 & 74 \\
\hline 1 & lpfc3 & DDN-tier4 & 63 \\
\hline 2 & en2 & mob1-en2 & 63 \\
\hline 2 & en3 & mob2-en2 & 63 \\
\hline 2 & en4 & mob3-en2 & 96 \\
\hline 2 & en5 & mob4-en2 & $554 \mathrm{MB} / \mathrm{s}$ \\
\hline
\end{tabular}


Then I tried the same thing with writes $501 \mathrm{MB} / \mathrm{s}$ with $28 \%$ CPU utilization.

RIO Saturation WRITE test

\begin{tabular}{|c|c|c|c|}
\hline PCI bus & Adapter & Device & Rate \\
\hline 1 & lpfc0 & DDN-tier1 & 57 \\
\hline 1 & $\operatorname{lpfc1}$ & DDN-tier2 & 54 \\
\hline 1 & lpfc2 & DDN-tier3 & 57 \\
\hline 1 & $\operatorname{lpfc3}$ & DDN-tier4 & 56 \\
\hline 2 & en2 & mob1-en2 & 62 \\
\hline 2 & en3 & mob2-en2 & 62 \\
\hline 2 & en4 & mob3-en2 & 62 \\
\hline 2 & en5 & mob4-en2 & 91 \\
\hline
\end{tabular}

Based upon the data rates observed we were indeed able to utilize the second RIO channel.

10. Saturate the I/O hub (or the $6 \mathrm{XX}$ bus connecting the $\mathrm{I} / \mathrm{O}$ hub to memory).

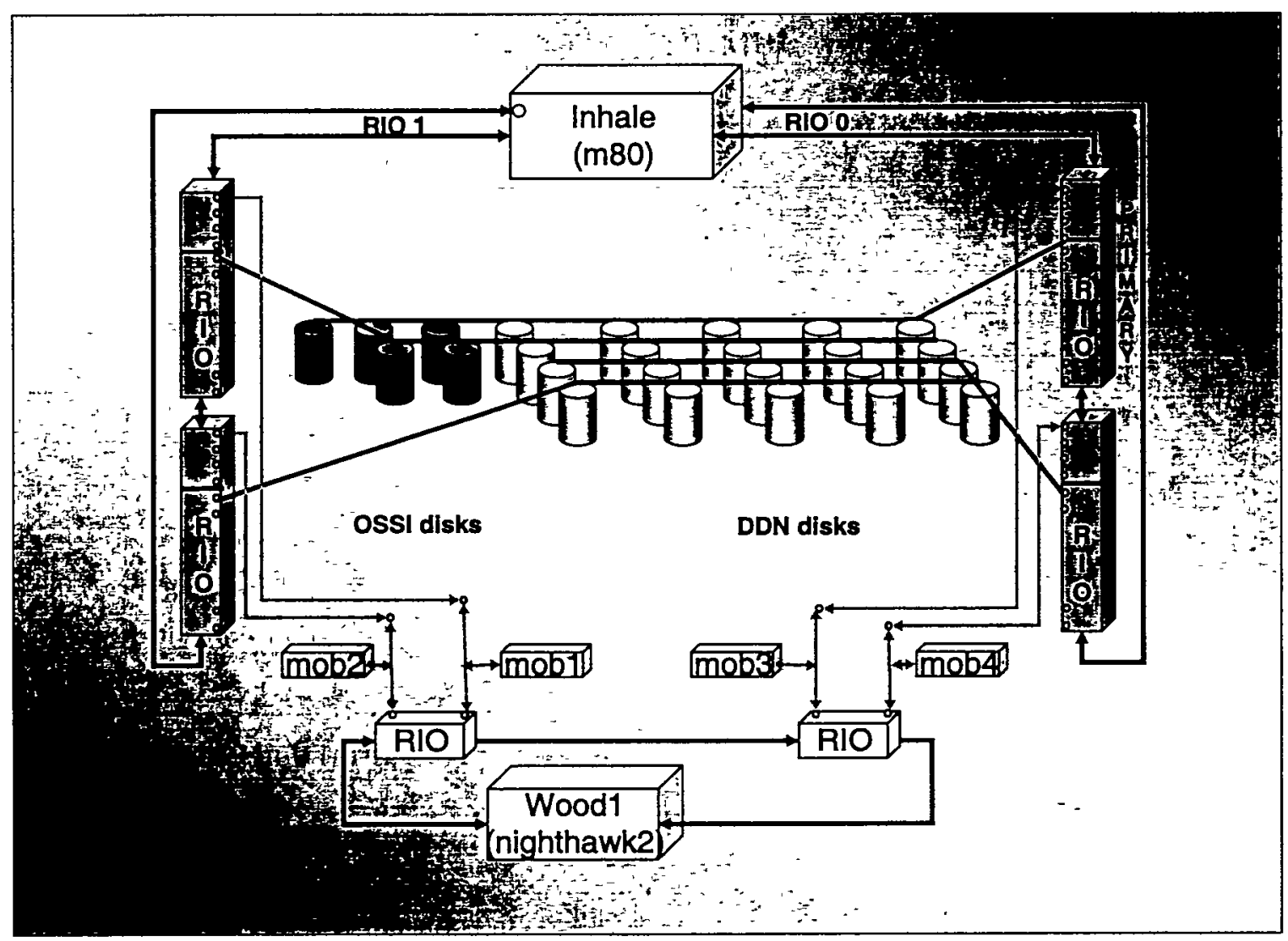

At this point I assumed that the second pair of RIO channels and RIO drawers would exhibit the same behavior as the pair I just tested. I distributed the 8 cards between the four RIO drawers, one card per PCI bus. Based upon previous testing, each card should be able to run at full speed in this configuration. Because each card is capable 
of running full duplex, theoretically we should be able to produce a load of $8 * 200 \mathrm{MB} / \mathrm{s}=1600 \mathrm{MB} / \mathrm{s}$ which should saturate the $6 \mathrm{XX}$ bus. Actually, we know that the emulex HBA will only run at a bit over $1 \mathrm{~GB} / \mathrm{s}$ full duplex on the M80.

Test 1: read four DDN disks, along with four gige writes to winterhawk nodes. Got $731 \mathrm{MB} / \mathrm{s}$ with $37 \%$ system time and $42 \%$ wait time.

\begin{tabular}{|c|c|c|}
\hline Interface & read & write \\
\hline $\operatorname{lpfc0}$ & 97 & - \\
\hline Ipfc1 & 97 & - \\
\hline $\operatorname{lpfc} 2$ & 97 & - \\
\hline $\operatorname{lpfc3}$ & 97 & - \\
\hline en2 & - & 91 \\
\hline en3 & - & 91 \\
\hline en4 & - & 70 \\
\hline en5 & - & 91 \\
\hline
\end{tabular}

Test2: read four DDN disks, read four nighthawk gige interfaces, write four winterhawk node gige interfaces. Got $782 \mathrm{MB} / \mathrm{s} 86 \%$ system time and $7 \%$ wait time.

Interface
\begin{tabular}{|c|c|c|}
\hline lpfc0 & 92 & read \\
\hline lpfc1 & 90 & - \\
\hline lpfc2 & 92 & - \\
\hline lpfc3 & 92 & - \\
\hline en2 & 45 & 58 \\
\hline en3 & 47 & 58 \\
\hline en4 & 47 & 58 \\
\hline en5 & 45 & 58 \\
\hline & 550 & $232=782$ \\
\hline
\end{tabular}

The disks ran pretty much as expected but the gige's were impacted. The same gige transfers performed without disk $\mathrm{I} / \mathrm{O}$ performed at around $75 \mathrm{MB} / \mathrm{s}$ each at $75 \%$ system time. 
Test3: Added two disk writes to Metastor disks ... writing 4 DDN disks, reading two metastor disks, reading four nighthawk2 gige's, writing four winterhawk gige's. Got $841 \mathrm{MB} / \mathrm{s}$ with $85 \%$ system time, $8.2 \%$ wait time.

\begin{tabular}{|c|c|c|}
\multicolumn{2}{|c}{ Interface } & read \\
\hline Ipfc0 & 45 & 63 \\
\hline lpfc1 & 45 & 63 \\
\hline lpfc2 & - & 75 \\
\hline lpfc3 & - & 74 \\
\hline en2 & 60 & 59 \\
\hline en3 & 60 & 59 \\
\hline en4 & 60 & 59 \\
\hline en5 & 60 & 59 \\
\hline & 330 & $511=841$ \\
\hline
\end{tabular}

Test4: Doubled the number of gigabit Ethernet data streams in each direction (8 each way). Got $934 \mathrm{MB} / \mathrm{s}$ with $100 \%$ system time. I/O was split $358 \mathrm{MB} / \mathrm{s}$ disk, $756 \mathrm{MB} / \mathrm{s}$ network.

\begin{tabular}{|c|c|c|}
\hline Interface & read & write \\
\hline $\operatorname{lpfc0}$ & 44 & 62 \\
\hline lpfc1 & 44 & 62 \\
\hline $\operatorname{lpfc2}$ & - & 73 \\
\hline $\operatorname{lpfc3}$ & - & 73 \\
\hline en2 & 70 & 81 \\
\hline en3 & 53 & 88 \\
\hline en4 & 70 & 81 \\
\hline en5 & 51 & 82 \\
\hline
\end{tabular}

The fact that the data rate increased shows that the $\mathrm{I} / \mathrm{O}$ hub and $6 \mathrm{XX}$ bus is not yet saturated. The fact that we are out of CPU power (without adding much useful bandwidth) shows that we've saturated the machine and hit the point on the curve where performance drops dramatically. None of our interfaces are running at full speed. 
11. Add four more cards (two gige NIC's and two emulex LP9000 HBA's) and again attempt to saturate the $\mathrm{I} / \mathrm{O}$ hub and $6 \mathrm{XX}$ bus. In the new configuration there's one physical RAID disk on each of the HBA's. I had to split two of the jumbo subnets to accommodate the additional gige interfaces.

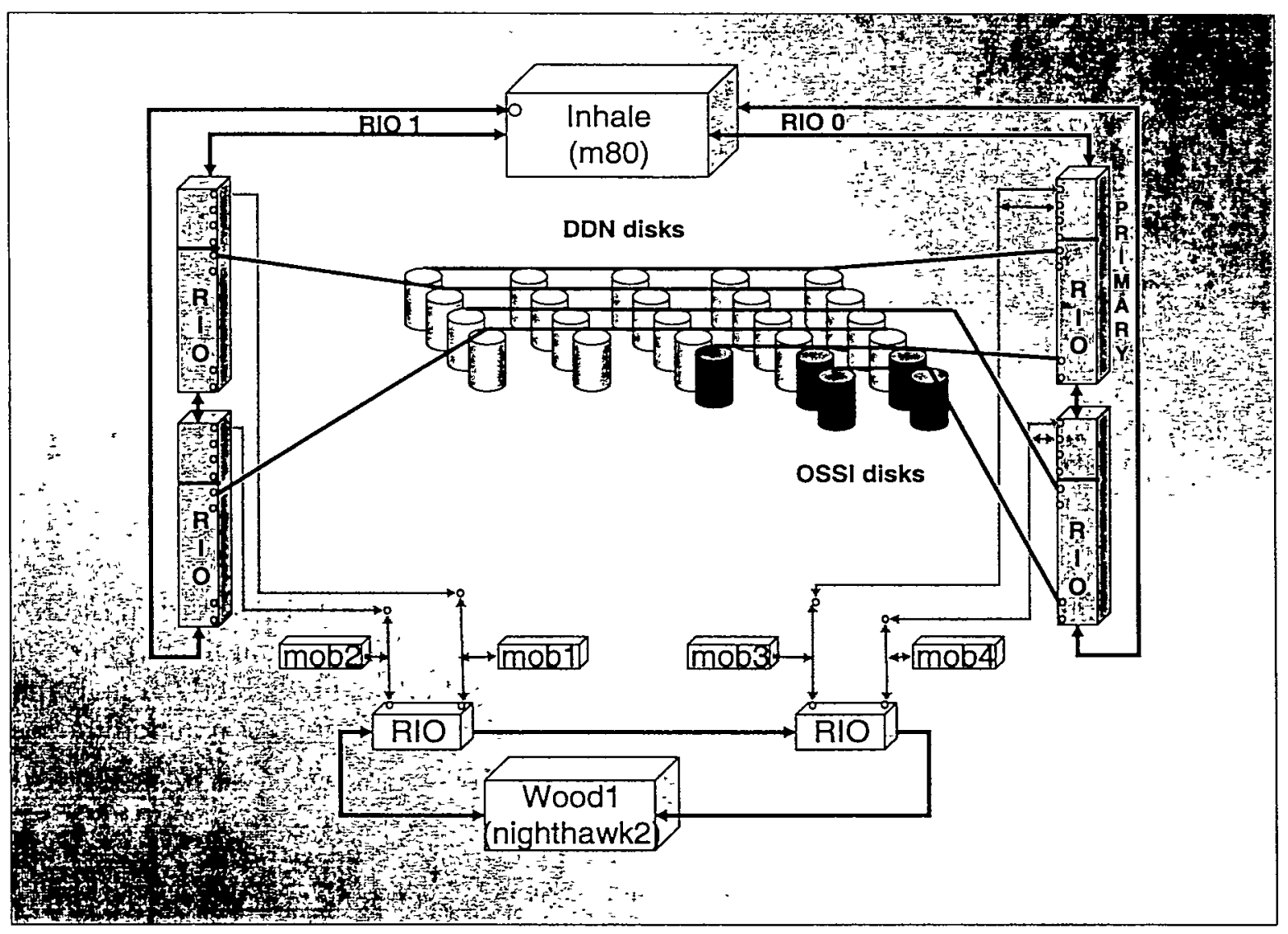


Test1: write four DDN disks, read 4 metastor, write two nighthawk nodes, write four winterhawk nodes. Got $1007 \mathrm{MB} / \mathrm{s}$ with $67 \%$ system time and $22 \%$ wait time. This test achieved the highest aggregate data rates I got during testing. Even tho the RIO load is unbalanced (668MB/s on RIO 1 compared to $339 \mathrm{MB} / \mathrm{s}$ on RIO 2) .. and most of the load was generated by writes $(844 \mathrm{MB} / \mathrm{s}$ write $163 \mathrm{MB} / \mathrm{s}$ read) all the devices are running close to full speed. This test leads me to believe we can possibly saturate the I/O capability of the machine using $6 \mathrm{M} 80$ processors rather than 8 .

\begin{tabular}{|c|c|c|}
\hline Interface & read & write \\
\hline lpfc0 & - & 72 \\
\hline lpfc1 & 82 & - \\
\hline lpfc2 & - & 71 \\
\hline lpfc3 & 81 & - \\
\hline lpfc4 & - & 74 \\
\hline $\operatorname{lpfc5}$ & - & 74 \\
\hline en2 & - & 90 \\
\hline en 3 & - & 91 \\
\hline en4 & - & 90 \\
\hline en5 & - & 91 \\
\hline en6 & - & 96 \\
\hline en 7 & - & 95 \\
\hline
\end{tabular}

I modified the previous test slightly by adding four gige reads (one on each gige interface) originating at the nighthawk node (wood1). I achieved an aggregate data rate of $1038 \mathrm{MB} / \mathrm{s}$ but the entire machine was saturated ( $99.9 \%$ system time).

Test2: read 6 disks while writing 6 gige interfaces. Got $887 \mathrm{MB} / \mathrm{s}$ with $69 \%$ system time and $21 \%$ wait time. However this is a VERY significant test. The only change from the previous test is that four of the disk devices are reading rather than writing! Note that lpfc4-5 and en4-5 are running at considerably higher data rates than the other interfaces. The four interfaces that are running at the higher speeds were on the SAME RIO loop. The other eight interfaces were all on the same RIO drawer. In this configuration all the devices on either RIO loop can run concurrently if the other loop is idle. But they won't run at full speed together! This is an indication of a bandwidth limitation in the $\mathrm{I} / \mathrm{O}$ hub or $6 \mathrm{XX}$ bus! This test doesn't seem make any sense at all. The I/O is more evenly distributed across the RIO buses and the read to write ratio is more evenly distributed yet performance has dropped off and one of the RIO loops appears to be saturated. Either of the RIO loops can run at full speed individually but they can't run together.

\begin{tabular}{|c|c|c|}
\hline Interface & read & write \\
\hline $\operatorname{lpfc0}$ & 75 & - \\
\hline $\operatorname{lpfc1}$ & 80 & - \\
\hline $\operatorname{lpfc2}$ & 77 & - \\
\hline lpfc3 & 81 & - \\
\hline
\end{tabular}




\begin{tabular}{|c|c|c|}
\hline lpfc4 & 96 & - \\
\hline lpfc5 & 96 & - \\
\hline en2 & - & 53 \\
\hline en3 & - & 53 \\
\hline en4 & - & 86 \\
\hline en5 & - & 86 \\
\hline en6 & - & 52 \\
\hline en7 & - & 52 \\
\hline
\end{tabular}


12. Distribute the I/O load evenly across the RIO loops and retest.

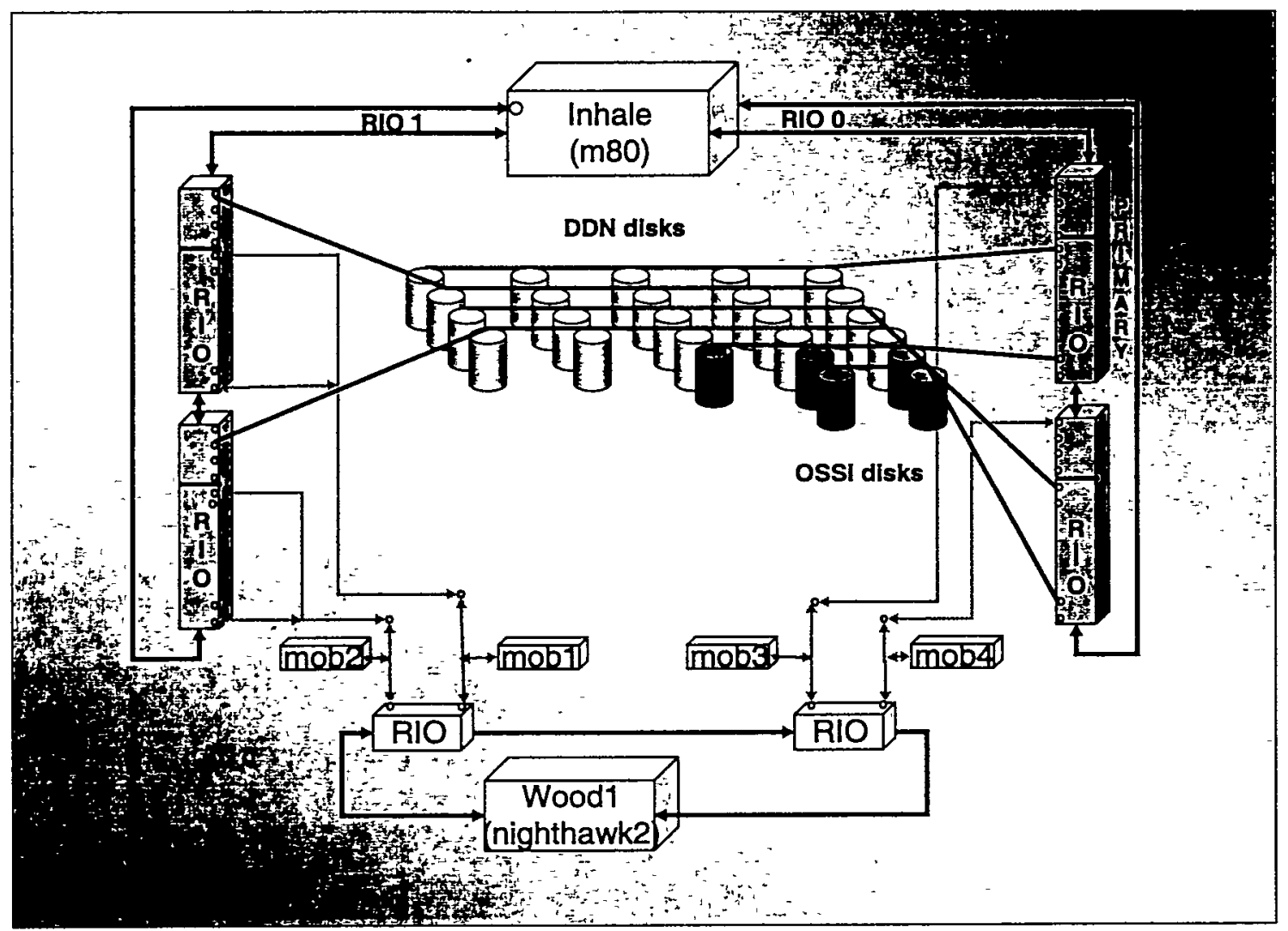

In this configuration we have three PCI cards in each RIO drawer with one card in each PCI bus (two cards on a single bus but separate bus repeaters).

Test1: Host read simulation. Read all four DDN disks (97MB/s each possible), read two metastor's ( $82 \mathrm{MB} / \mathrm{s}$ possible) while concurrently writing to three winterhawk gige's (en3-5, one winterhawk was down at this point), and three gige interfaces on the nighthawk node (en2,en6,en7).

We achieved $902 \mathrm{MB} / \mathrm{s}$ with $76 \%$ system load and $16 \%$ wait.

Interface
\begin{tabular}{|c|c|c|}
\hline lpfc0 & 81 & write \\
\hline Ipfc1 & 88 & - \\
\hline lpfc2 & 80 & - \\
\hline lpfc3 & 81 & - \\
\hline lpfc4 & 88 & - \\
\hline lpfc5 & 81 & - \\
\hline en2 & - & 64 \\
\hline en3 & - & 63 \\
\hline en4 & - & 70 \\
\hline en5 & - & 70 \\
\hline en6 & - & 70 \\
\hline
\end{tabular}


Interface

read

write

\begin{tabular}{|c|c|c|}
\hline Ipfc0 & - & 72 \\
\hline $\operatorname{lpfc1}$ & 82 & - \\
\hline $\operatorname{lpfc2}$ & - & 69 \\
\hline $\operatorname{lpfc3}$ & 81 & - \\
\hline $\operatorname{lpfc4}$ & - & 72 \\
\hline $\operatorname{lpfc5}$ & 94 & - \\
\hline en2 & - & 87 \\
\hline en3 & 84 & - \\
\hline en4 & - & 99 \\
\hline en5 & 83 & - \\
\hline en6 & - & 86 \\
\hline en7 & 78 & - \\
\hline & 502 & $485=987 \mathrm{MB} / \mathrm{s}$ \\
\hline
\end{tabular}

13. At this point we are able to saturate the I/O capability of the system. We want to find out how much CPU power is required to saturate the system's I/O capabilities. Using the system's service processor capabilities we disable one cpu at a time and rerun our tests until aggregate $\mathrm{I} / \mathrm{O}$ throughput degrades.

Early tests show that if we add additional gige streams, we saturate the CPU capability of the machine. However in the optimal configuration tested above with six gige streams there's CPU left. Adding additional I/O streams (which requires more CPU) does not improve the overall $\mathrm{I} / \mathrm{O}$ throughput significantly. Individual gigabit Ethernet tests show that it requires almost a dedicated CPU to maintain a full bandwidth transfer. The I/O capability of the machine can be saturated by the 8 CPU's as demonstrated by the fact that all I/O streams are degraded equally in the preceding tests. Note that even with 8 processors per M80 the ratio of $1 / O$ capabilityto-processor is significantly lower than with the winterhawks (see following winterhawk test results). With the winterhawks we achieved $280 \mathrm{MB} / \mathrm{s}$ with 4 processors. With the $\mathrm{M} 80$ we can achieve $980 \mathrm{MB} / \mathrm{s}$ with 8 processors $(70 \mathrm{MB} / \mathrm{s}$ per processor on winterhawk compared to $122 \mathrm{MB} / \mathrm{s}$ per processor with the M80). However, since the M80 can be purchased with 6 CPU's (one four processor module and one two processor module) we could consider a six processor configuration if the savings are significant.

(SHOULD TEST with 6 CPU's didn't realize we could get a 2 CPU module until recently) .... 


\section{HPSS Based Testing (Jim Daveler)}

At this point we will have a pretty good idea of the $\mathrm{I} / \mathrm{O}$ capabilities of the current test configuration. We will configure the system as an HPSS mover and verify system behavior running under the actual storage application.

Since we do not have sufficient tape bandwidth in the I/O testbed to stress a system of this capability, we will simulate the HPSS migration load through host read operations. Since the same path (disk device to/from gige interface) will be used for tape access a host read or write will provide a valid simulation.

The preceding simulations have shown that the hardware should be able to support six concurrent (nearly) full speed data streams. Our challenge is to configure the HPSS system in a fashion that causes all the interfaces will be used. Our goal is to be able to exploit the parallelism inherent in HPSS without undue administrative complication. To the best of my knowledge, no HPSS site has configured a system running only movers on a platform with the power of the M80 and having the network bandwidth provided by our I/O testbed. A complete HPSS system was configured on an IBM S80 at ORNL but they did not have as many I/O cards or a robust multi-subnet gigabit Ethernet infrastructure.

An HPSS mover facilitates data transfers between storage clients, other HPSS movers and devices. We must therefore insure that both the clients and other HPSS movers spread their data transfers across all six gigabit interfaces available on the M80. To accomplish this goal, we configured six HPSS movers on the M80 and set the HPSS "data-host" address for each mover to a different M80 gigabit Ethernet interface. This configuration insures that when another HPSS mover transmits data to the M80 the M80 interface used will be determined by the destination data mover process. Because our current production HPSS movers all have their "data host" addresses set to their SP switch IP address we must assign hard routes on the M80 to handle the situation. The hard route on the M80 will be assigned to the production mover's jumbo gigabit Ethernet address. Because the existing HPSS data movers are distributed across the center's four jumbo subnets, this assignment will naturally distribute the data flow between the M80 and the existing HPSS data movers across at least four of the M80's gigabit interfaces. Data movement between the storage clients and the data mover processes is parallelized by the HPSS client logic which allows a storage client to specify which client interfaces are available for a transfer. This logic will distribute the load across the specified interfaces. Because the M80 will have six interfaces and we currently have only four jumbo gige subnets, we will have to split two of the jumbo subnets on the M80 side to accommodate the additional interfaces. Use of IP aliases and thoughtful initial assignment of IP addresses can help to distribute the load across six jumbo subnets rather than four. Until our data center increases the number of gigabit Ethernet subnets, two of the M80 gigabit Ethernet interfaces are likely to be underutilized and care should be exercised in the HPSS configuration to insure that two HPPS data stripes are not 
assigned to the same jumbo subnet. An easy way to avoid problems is to establish an HPSS administrative convention that stripes are assigned only to the first four movers on an M80.

While running these tests we also discovered that AIX can not read a file system at much more than $200 \mathrm{MB} / \mathrm{s}$ (even when the data is generated in memory - reading from /dev/zero or a sparse file!).

1. Configure HPSS devices.

Because the LLNL production environment currently supports four jumbo gigabit interfaces, the optimum (widest possible) stripe width is 4 . We configured a fourwide striped disk on M80 movers 1-4 and two single-wide disks on mover5 and mover6. With this configuration we should be able to demonstrate individual disk transfer rates, striped transfer rate, and aggregate (mover saturated) transfer rates. The four-wide stripe was configured on the DDN disk and the individual HPSS volumes were configured on the two metastor disks. We originally configured virtual volume sizes of $32 \mathrm{MB}$ but reconfigured the four way striped $\mathrm{COS}$ and one unstriped COS with an $8 \mathrm{MB} \mathrm{VV} \mathrm{(virtual} \mathrm{volume)} \mathrm{size} \mathrm{in} \mathrm{an} \mathrm{attempt} \mathrm{to} \mathrm{increase}$ performance. PFTP performance was not affected significantly by the VV size however HSI performance improved. Network and operating system parameters used during the HPSS simulation testing were used for this test. In the following tests, the HPSS core servers were running on one winterhawk node and the HPSS database (Transarc SFS) was running on another winterhawk nodes. I suspect that the write rates suffered due to the fact SFS was not running on the same physical hardware. Other HPSS testing has not shown severe discrepancies between read and write rates.

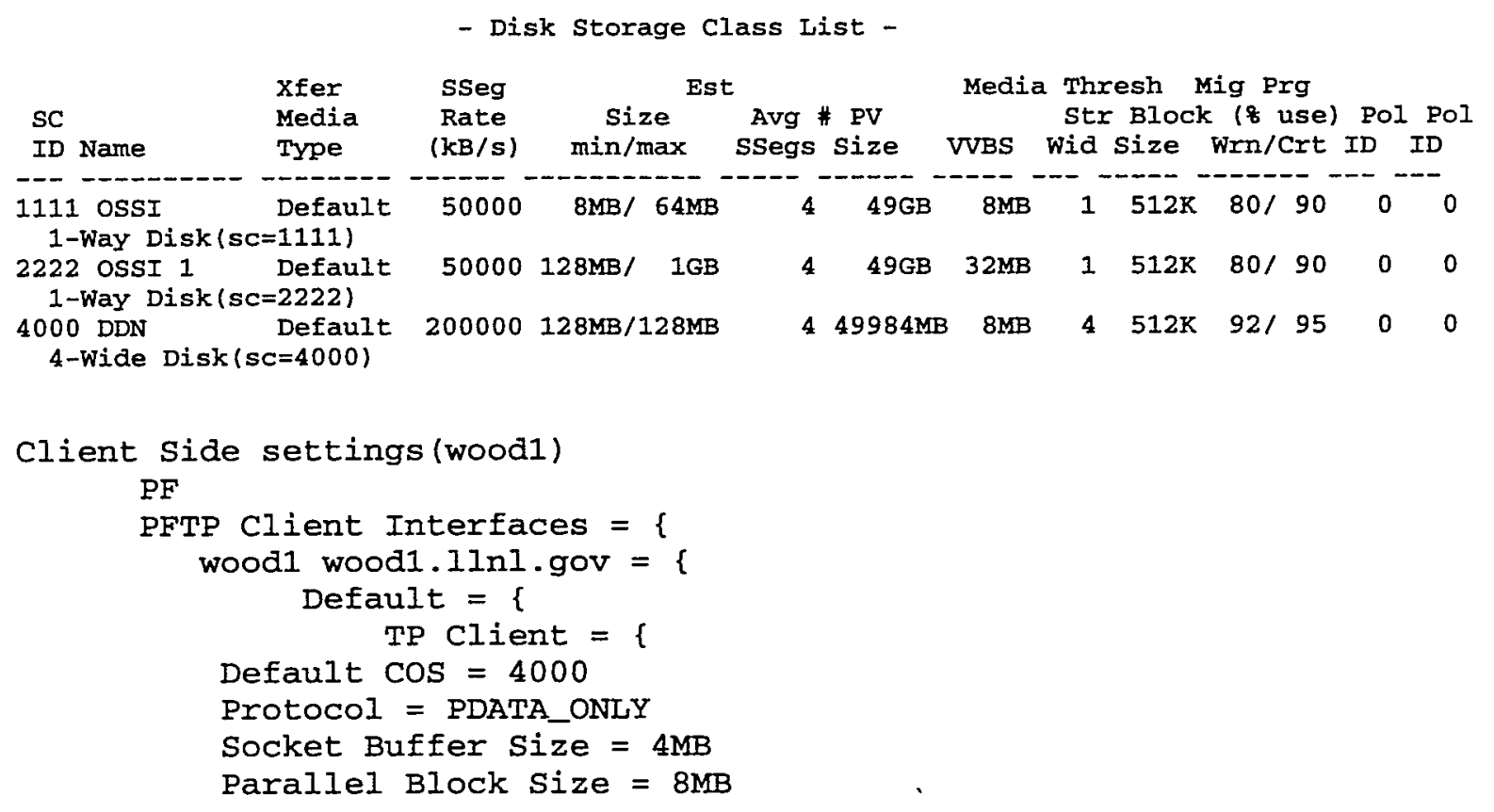




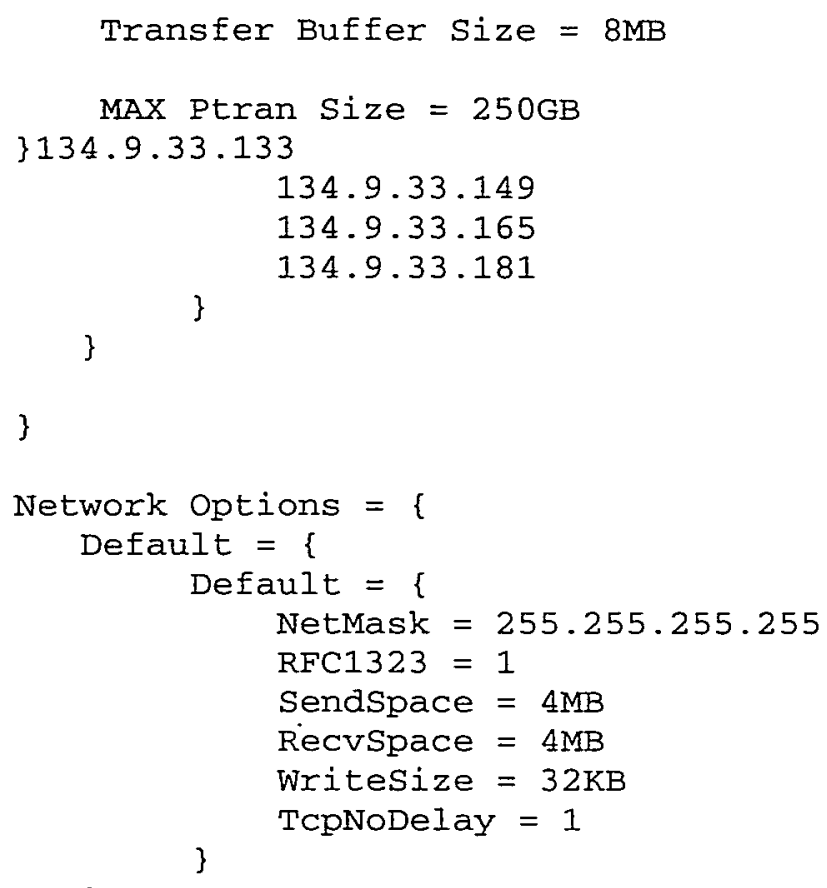

2. Test data rate to an individual HPSS volume.

We tested individual data rates by reading and writing one of the un-striped classes of service (2222) from the mob4 "winterhawk" system.

\begin{tabular}{|c|c|}
\multicolumn{1}{|c|}{ Operation } & Data Rate $(\mathrm{MB} / \mathrm{s})$ \\
\hline read & 75 \\
\hline write & 41 \\
\hline
\end{tabular}

3. Test concurrent data rates to two un-striped classes of service.

\begin{tabular}{|c|c|c|c|} 
Operation & \multicolumn{1}{c}{$\begin{array}{c}\text { mob4 } \\
(\mathrm{MB} / \mathrm{s})\end{array}$} & $\begin{array}{c}\text { mob3 } \\
(\mathrm{MB} / \mathrm{s})\end{array}$ & $\begin{array}{c}\text { Total } \\
(\mathrm{MB} / \mathrm{s})\end{array}$ \\
\hline read & 72 & 72 & 144 \\
\hline write & 40 & 40 & 80 \\
\hline
\end{tabular}

4. Test data rate to a striped HPSS volume.

We read and wrote the 4 way class of service from the nighthawk system. 
Operation read
Data Rate (MB/s)

write

140 
Test aggregate (all six movers active) data rate.

\begin{tabular}{|c|c|c|c|c|}
\hline Operation & $\begin{array}{c}\text { mob4 } \\
(\mathrm{MB} / \mathrm{s})\end{array}$ & $\begin{array}{l}\text { mob3 } \\
(\mathrm{MB} / \mathrm{s})\end{array}$ & $\begin{array}{l}\text { wood } 1 \\
(\mathrm{MB} / \mathrm{s})\end{array}$ & $\begin{array}{l}\text { Total } \\
(\mathrm{MB} / \mathrm{s})\end{array}$ \\
\hline read & 70 & 70 & 203 & 343 \\
\hline write & 40 & 40 & 142 & 222 \\
\hline
\end{tabular}




\section{HSI data rates (Mike Gleicher)}

From: Mike Gleicher <gleicheretoofast10.1Inl.gov>

To: jdaveleretoofast10.1lnl.gov, kfitz@llnl.gov, mkgesan.rr.com subject: wood1 - slightly better results

Jim/Keith -

Here are a couple of tests I just ran from wood1 -

$\mathrm{M}:[$ nmob2] /home/jdaveler->get /dev/nul] : sparse.hoho

get /dev/null : /home/jdaveler/sparse.hoho (2002/02/13 17:15:38

2147483647 bytes, 223554.9 kBS )

M: [nmob2]/home/jdaveler->get /dev/nul] : sparse.hoho

get /dev/null : /home/jdaveler/sparse.hoho (2002/02/13 17:15:38

2147483647 bytes, 303720.6 KBS )

Date: Wed, 13 Feb 2002 19:59:23 -0800

\section{LLNL Testbed}

HSI on wood1, 4 jumbo-frame gige adaptors

4 way disk stripe on (??) - 4 jumbo frame gige

adaptors

16MB mover buffer
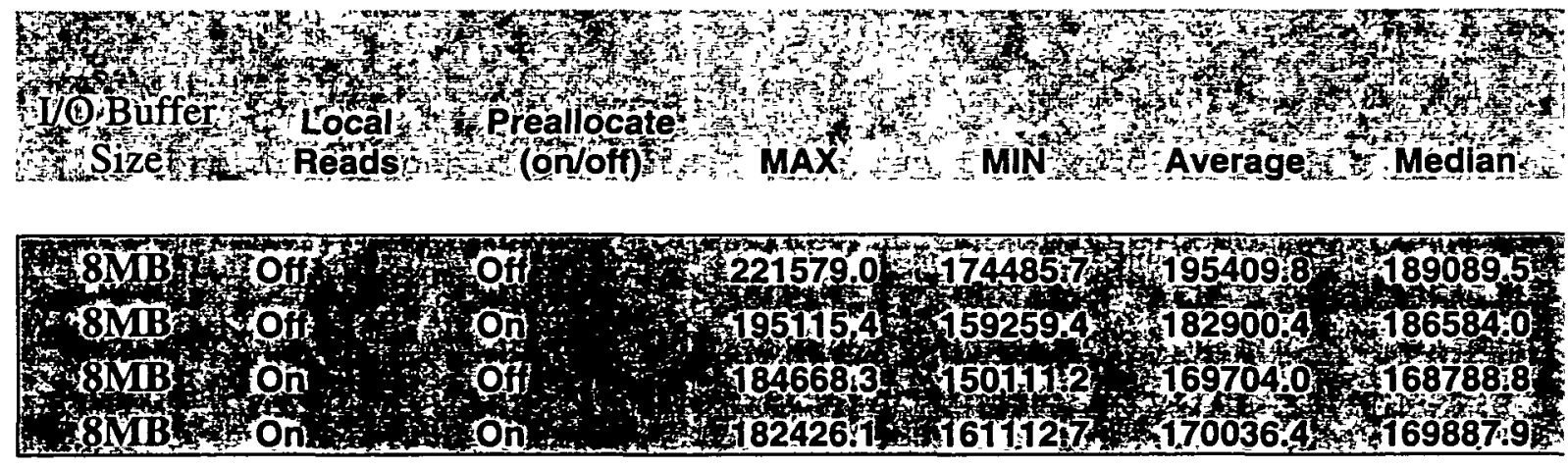

\begin{tabular}{|c|c|c|c|c|c|c|}
\hline $\begin{array}{r}16 \mathrm{MB} \\
16 \mathrm{MB} \\
16 \mathrm{MB} \\
16 \mathrm{MB} \\
16 \mathrm{M} \\
1 \%\end{array}$ & $\begin{array}{l}\text { Offy } \\
\text { Off } \\
\text { On } \\
\text { On } \\
\text { On }\end{array}$ & $\mathrm{offl}^{2+2}$ & $\begin{array}{r}212001.7 \\
229066.8 \\
190776.1 \\
180873.6\end{array}$ & $\begin{array}{l}164989: 7 \\
187135: 0 \\
1566417 \\
156067.4\end{array}$ & $\begin{array}{l}1937072 \\
2074267 \\
171912 ; 9 \\
168496.9\end{array}$ & $\begin{array}{l}1961578 \\
2050768 \\
1704580 \\
168478.2\end{array}$ \\
\hline $24 M B$ & Off & Off & 228224.7 & 166164.0 & 201116.8 & 202070.0 \\
\hline $24 M B$ & Off & On & 213710.3 & 174740.1 & 191644.9 & 188145.2 \\
\hline $24 M B$ & On & Off & 175475.2 & 133465.4 & 157693.2 & 155952.7 \\
\hline 24MB & On & On & 166382.3 & 130454.1 & 158582.7 & 163889.7 \\
\hline
\end{tabular}




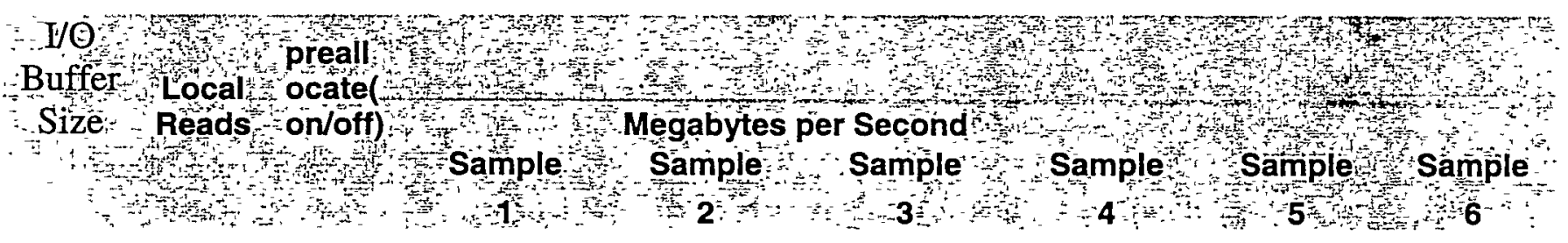

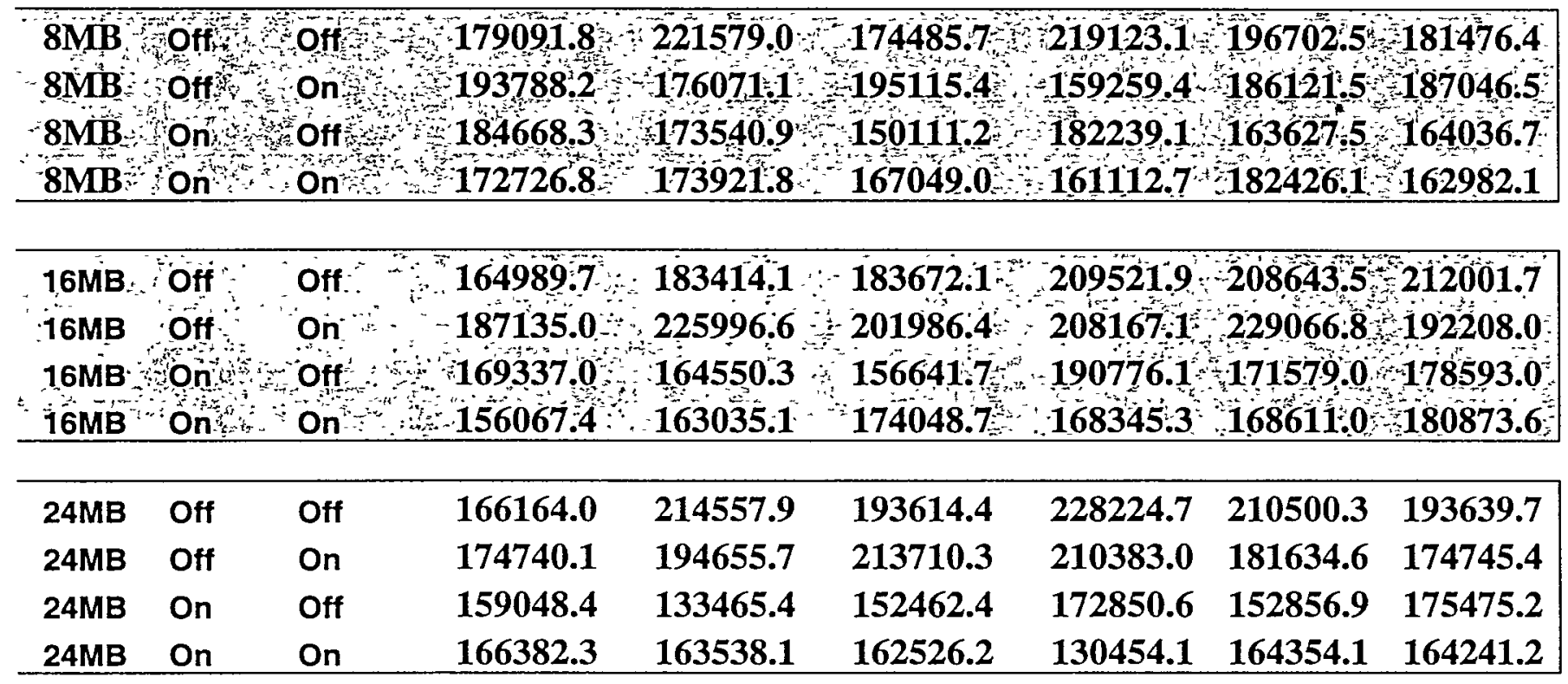




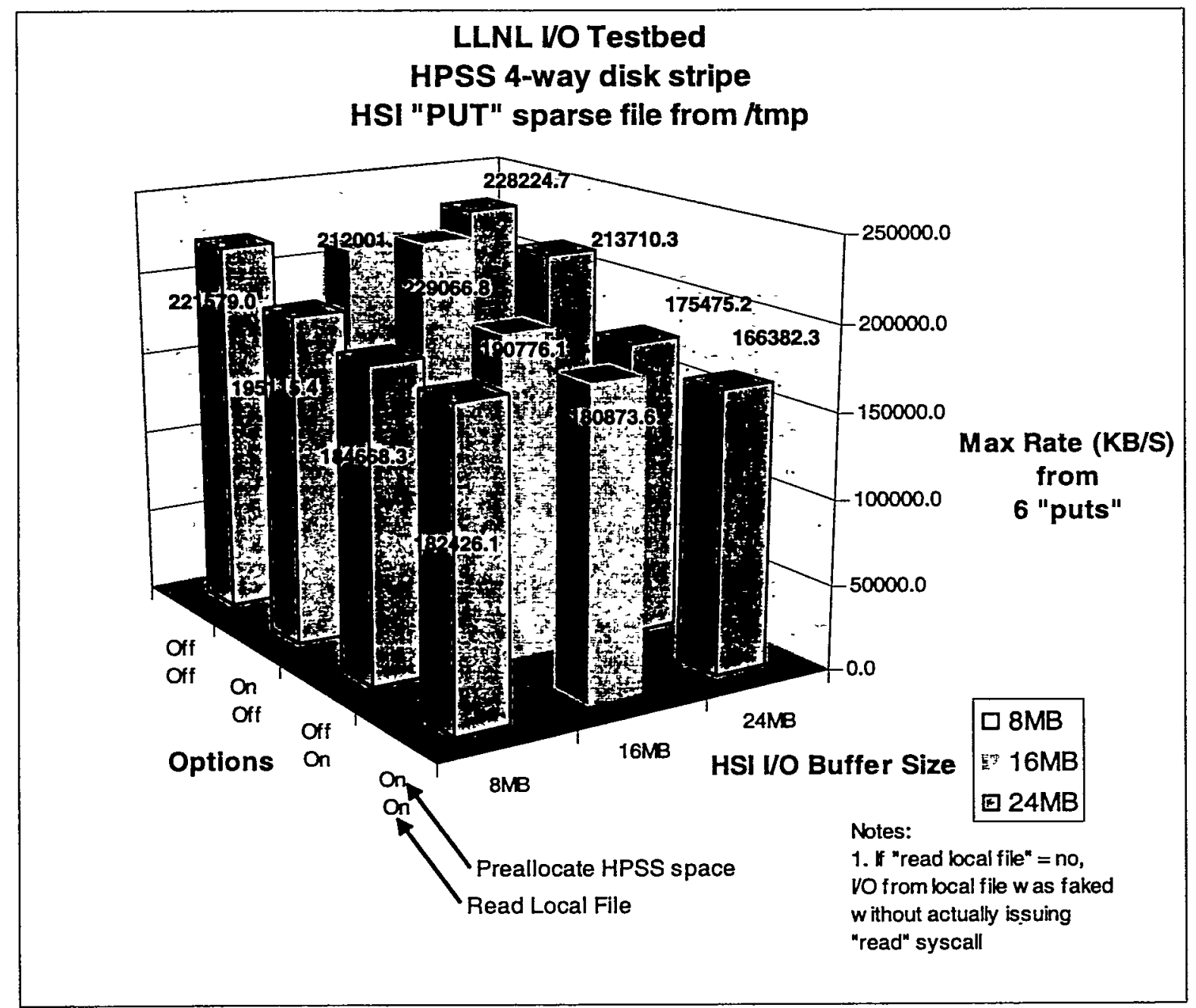




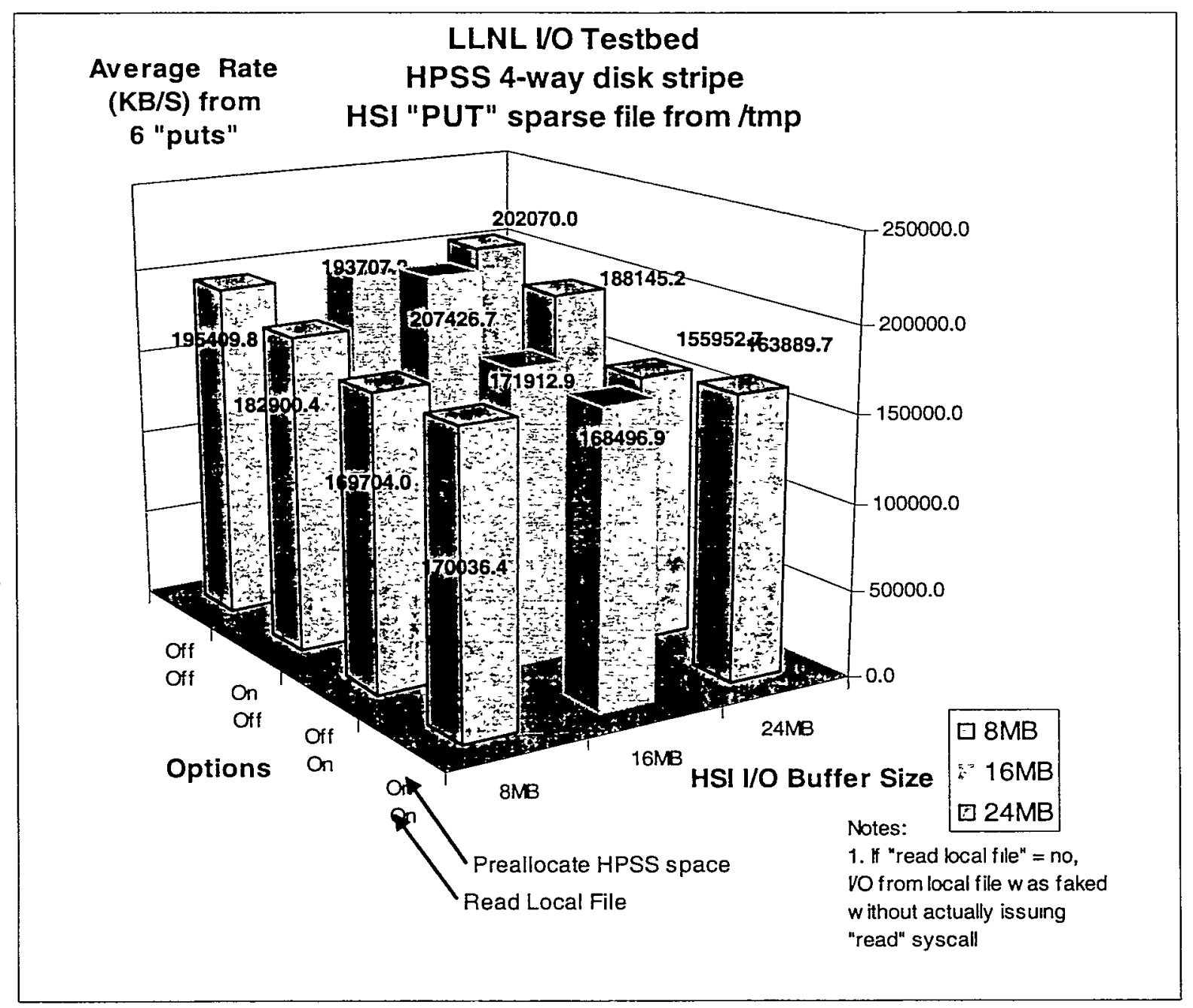




\section{Six Processor testing}

Processors are expensive $(\sim 25 \mathrm{~K}$ each $)$ ! HPSS simulations have shown that the $\mathrm{I} / \mathrm{O}$ bandwidth can be saturated at around $75 \% \mathrm{cpu}$ utilization. In this test I went into the service processor menu and turned off the last two processors (6 and 7). I then reran the HPSS simulation tests.

1. HPSS read simulation: achieved a total of $874 \mathrm{MB} / \mathrm{s}$

\begin{tabular}{|c|c|c|}
\multicolumn{1}{c}{ Interface } & read & write \\
\hline lpfc0 & 83 & - \\
\hline lpfc1 & 80 & - \\
\hline lpfc2 & 83 & - \\
\hline lpfc3 & 81 & - \\
\hline lpfc4 & 90 & - \\
\hline lpfc5 & 90 & - \\
\hline en2 & - & 70 \\
\hline en3 & - & 75 \\
\hline en4 & - & 74 \\
\hline en5 & - & 74 \\
\hline en6 & - & 74 \\
\hline & 507 & 367
\end{tabular}

2. HPSS write simulation: achieved a total of $726 \mathrm{MB} / \mathrm{s}$

\begin{tabular}{|c|c|c|}
\hline Interface & read & write \\
\hline $\operatorname{lpfc0}$ & - & 64 \\
\hline Ipfc1 & - & 66 \\
\hline $1 \mathrm{pfc} 2$ & - & 39 \\
\hline lpfe3 & - & 65 \\
\hline $\operatorname{lpfc} 4$ & - & 78 \\
\hline lpfc5 & - & 79 \\
\hline en2 & 68 & - \\
\hline en3 & 67 & - \\
\hline en4 & 67 & - \\
\hline en5 & 67 & - \\
\hline en6 & 60 & - \\
\hline en7 & 60 & - \\
\hline
\end{tabular}




\section{SP Attached M80 Analysis (Todd Heer)}

Certain functions must be present to enable a machine to be managed and administered like the storage groups current SP nodes. This requirement gives continuity of administration for new machines, ensuring that they can benefit from the commands, tools, and functionality already present. We must be mindful to not linearly add administrative overhead for each new machine introduced.

This functionality is given when the M80 is attached to the control workstation via a cable which interfaces to the M80 server via the PCI Card for SP Control Workstation Attachment (feature \#3154). Internally, this pci card is placed in slot 7 of the primary $\mathrm{I} / \mathrm{O}$ drawer of the M80 and a cable is run from the card to a specific connector on the planar board. The test was performed on AIX 433 maintenance level 8 with PSSP version 3.2, including the requisite APAR IY16350.

Here is what I found:

1) Can the $M 80$ be powered off and on using SP utilities?

Yes. Much like any normal (in-frame) SP node, the hardmon daemon does control power to the M80 through tools such as spmon. This was verified.

2) Does the SP console (sIterm) perform properly?

Yes. This is often accessed by executing spmon -open node $<X>$. This is where we had some issues initially, however once the console was changed to /dev/tty0 on the M80 it worked fine. This likely would not have been an issue if we had installed the M80 from the cws directly initially (rather than retrofitting it to the $\mathrm{SP}$ - which is a good way to learn how things work).

3) Does SP software run correctly on the M80 as an integrated environment?

Yes, as far as we tested. I was able to get host responds up, which means the High Availability Infrastructure was operating normally. There was no high performance switch connection, so switch responds was not tested.

4) Did Kerberos authentication work?

N/A. This was not tested. I surmise, based on what I did test, that indeed it would work as designed. Again, we retrofitted an already install standalone M80 into an existing SP environment. Had we successfully installed from scratch, the proper Kerberos files would have been sent to the client (M80) from the cws.

5) Does a network install perform properly?

This can be answered in two parts. First, there are the mechanics of the install. Those being a network boot, successful transmission via bootp and tftp of a boot image, nim install beginning, mksysb image being transferred over the network, and having it installed to disk on the client. This part worked. I did encounter an issue which went unresolved for the last part of a successful node install, the 
customize portion. Given time restrictions, I was not able to determine why the customize hung at a led, but having debugged many SP node customizations, I can safely say that this would not be a problem. I assume there was a permissions problem with a config file or perhaps a subset of a network problem. The important thing is that for the purposes of a network install, the machine behaved exactly like a normal SP node. In that sense, the install did perform properly.

\section{Oustanding Issues}

Having only one of these systems on hand to test, we can't verify the scalability of two or more systems. Presumably we would add systems over time, as we augment HPSS movers and our throughput needs increase. What we don't know is the effect of several of these machines on a control workstation and what the limit is. This question would have to be answered within some acceptable margin of error before choosing to go down this path. IBM development/support could be consulted on this issue.

Todd Heer

Data Storage Group

LLNL

theer@llnl.gov 


\section{Conclusions}

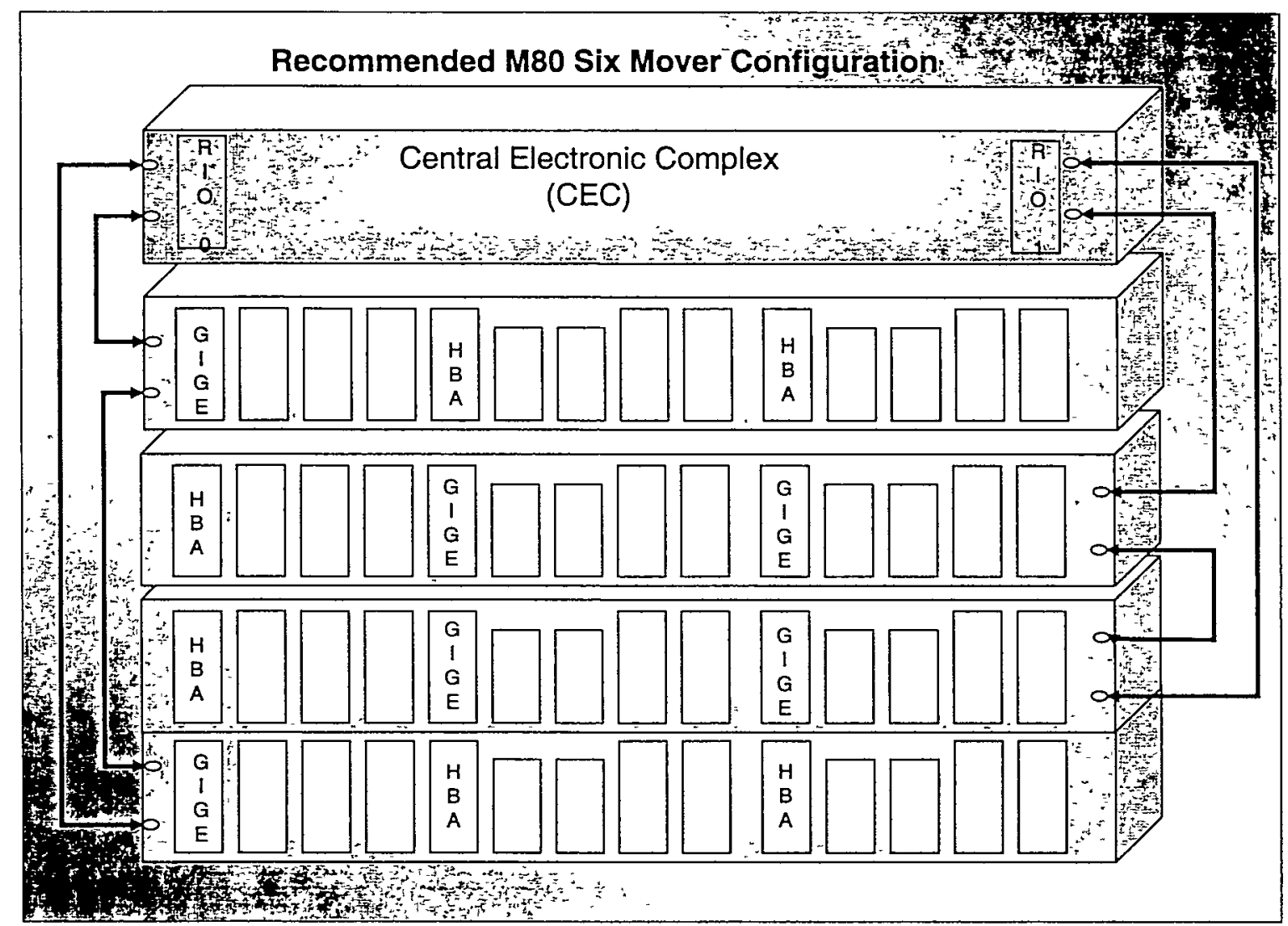

1. You can achieve a data rate of around $320 \mathrm{MB} / \mathrm{s}$ on the $64 \mathrm{bit} 66 \mathrm{MHz} \mathrm{PCI}$ bus which can run at a theoretical rate of $528 \mathrm{MB} / \mathrm{s}$ (around $60 \%$ of theoretical). This translates to the fact that you should install no more than two full duplex $100 \mathrm{MB} / \mathrm{s} \mathrm{PCI}$ cards (like IBM gige's or emulex HBA's) in each PCI bus.

2. You can achieve a data rate of around $375 \mathrm{MB} / \mathrm{s}$ on a RIO connection which has a $500 \mathrm{MB} / \mathrm{s}$ theoretical transfer rate. (around $75 \%$ theoretical). This means that either of the two PCI buses could nearly saturate a RIO connection if you installed four cards in the PCI bus. However the RIO speed isn't a limitation if you adhere to rule 1 ... only two cards in a PCI bus. This means only four cards in a RIO drawer in which case there's enough RIO bandwidth (375MB/s in each direction) to handle all four cards.

3. An individual RIO drawer is bound to a single RIO connection on the $/ / O$ hub card. This limits an individual RIO drawer to $375 \mathrm{MB} / \mathrm{s}$ in each direction. This means that you must do a mixture of reads and writes in order to utilize the total bandwidth offered by the two PCI buses in a RIO drawer. 
4. A pair of RIO drawers have a potential data rate of $750 \mathrm{MB} / \mathrm{s}(375 \mathrm{MB} / \mathrm{s}$ in each direction) if it was configured with 2 cards per bus and therefore four cards per RIO drawer.

5. Four fully loaded RIO drawers could provide an aggregate bandwidth of $1500 \mathrm{MB} / \mathrm{s}$ BUT ... the I/O hub module or $6 \mathrm{XX}$ memory bus saturates at around $1000 \mathrm{MB} / \mathrm{s}$.

6. In order for data to "stream" through the M80 the data must traverse the I/O subsystem twice. Once as the data is written into the M80 memory and once as the data is read out of the M80 memory. To move 100MB/s of data through a mover requires $200 \mathrm{MB} / \mathrm{s}$ of mover bandwidth. It seems reasonable to assume that each data stream through the mover can achieve a data rate of $80 \mathrm{MB} / \mathrm{s}$ (when we get the emulex HBA's fixed). Assuming that the M80 is capable of handling $1000 \mathrm{MB} / \mathrm{s}$ that comes out to a bit over 6 concurrent data streams. Six streams implies 12 cards. However since each card is capable of full duplex operation 12 cards effectively over allocates the M80 by a factor of two.

7. If you assume that you only get $60 \mathrm{MB} / \mathrm{s}$ per stream, you can configure 8 movers $(60 \mathrm{MB} / \mathrm{s} * 8 \mathrm{streams}=480 \mathrm{MB} / \mathrm{s})$ on the $\mathrm{M} 80$. You actually require $2 * 480 \mathrm{MB} / \mathrm{s}$ because data must traverse both the NIC and HBA for a total of $960 \mathrm{MB} / \mathrm{s}$.

8. I was not able to read an AIX file system at much more than $200 \mathrm{MB} / \mathrm{s}$ even when reading from /dev/zero or a sparse file. I also suspect that you can not write to GPFS from an individual node at much over that rate. I could not test this theory on the I/O testbed Nighthawk system because the SSA based GPFS system was only designed to deliver $100 \mathrm{MB} / \mathrm{s}$. However, if this is theory is valid it doesn't make sense to stripe more than three disks capable of running at $80 \mathrm{MB} / \mathrm{s}$ because the client can not deliver the data fast enough. 


\section{Appendix A: Network options}

$<$ > no -a

extendednetstats $=0$

thewall $=1048496$

sockthresh $=85$

sb_max $=2097152$

somaxconn $=1024$

clean_partial_conns $=0$

net_malloc_police $=0$

rto_low $=1$

rto_high $=64$

rto_limit $=7$

rto_length $=13$

inet_stack_size $=16$

axptab_bsiz $=7$

arptab_nb $=25$

tcp_ndebug $=100$

ifsize $=35$

axpqsize $=5$

ndpqsize $=50$

route_expire $=5$

send_file_duration $=300$

fasttimo $=200$

routerevalidate $=0$

nbc_limit $=786352$

nbc_max_cache $=131072$

nbc_min_cache $=1$

nbc_pseg $=0$

nbc_pseg_Iimit $=16777192$

strmsgsz $=0$

strctlsz $=1024$

nstrpush $=8$

strthresh $=85$

psetimers $=20$

psebufcalls $=20$

strturncnt $=15$

pseintrstack $=12288$

lowthresh $=90$

medthresh $=95$

psecache $=1$

subnetsarelocal $=1$

maxtt $I=255$

ipfragtt $1=60$

ipsendredirects $=1$

ipforwarding $=0$

udp_ttl $=30$

tcp_tt $1=60$

arpt_killc $=20$

tcp_sendspace $=655360$

tcp_recvspace $=655360$

udp_sendspace $=655360$

udp_recvspace $=655360$

rfc1122addrchk $=0$

nonlocsrcroute $=1$

tcp_keepintvI $=150$ 


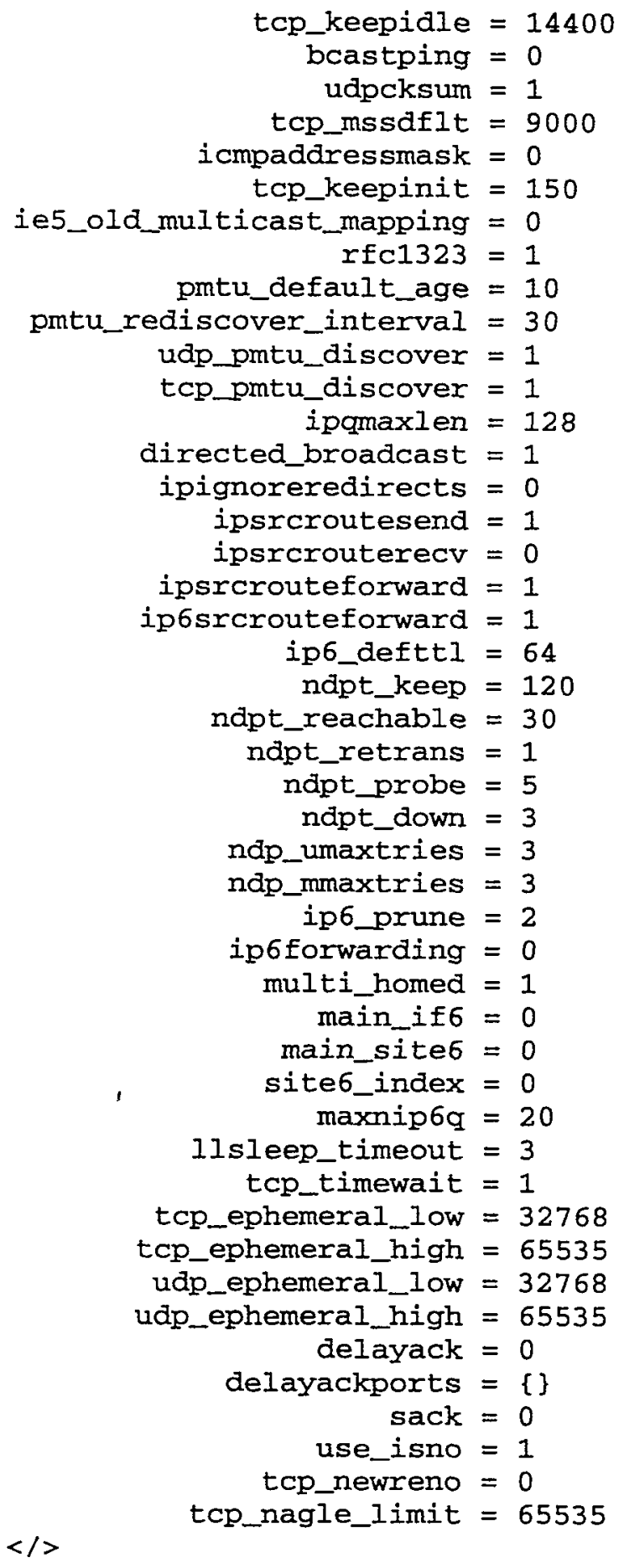




\section{Appendix B: Outstanding Issues}

1. For some reason, the cisco 6509 disables the $100 \mathrm{Mb} / \mathrm{s}$ Ethernet interface built into the primary RIO drawer when the system goes down. "interface show status" on the cisco shows the link in err-disable state.

Setting the following options on the csico seems to mitigate the problem:

"errdisable recovery cause link-flap"

"errdisable recovery interval 30"

2. The emulex LP8000 and LP9000 adapters lockup occasionally when volume groups are being varied online. Using the emulex diagnostic "lputil" to reset the adapter unhangs the adapter (most of the time).

We are working with emulex to resolve the issue.

3. When you install multiple HBA's on a single PCI bus using the M80 hot swap logic and then run "cfgmgr" to detect and configure the devices only the first HBA on the channel is detected. The other HBA(s) give "bus conflict" error messages if you examine the verbose "cfgmgr" output.

Running "cfgmgr - pl" gets around the problem. Emulex will fix the problem in a later release.

4. The LP8000 and LP9000 adapters do not write as fast as on the IBM "winterhawk2" nodes. We can achieve write rates of $96 \mathrm{MB} / \mathrm{s}$ on winterhawk nodes we only get around $80 \mathrm{MB} / \mathrm{s}$ from the $\mathrm{M} 80$. System, driver, and HBA microcode levels are the same.

We are working with emulex to resolve the issue. It would be interesting to test the IBM supported version of the emulex LP9000.

5. Concurrent read/write rates on the emulex LP8000 and LP9000 adapters are low. We only achieved $112 \mathrm{MB} / \mathrm{s}$ on concurrent read/writes on the M80. We can achieve 150$160 \mathrm{MB} / \mathrm{s}$ on the winterhawk2 nodes and this rate may be limited by the $64 \mathrm{bit} 33 \mathrm{MHz}$ PCI bus which only has a theoretical data rate of $264 \mathrm{MB} / \mathrm{s}$.

We are working with emulex to resolve the issue. Testing the IBM version of the LP9000 would be interesting.

6. TCP/IP over fibre channel using LP8000 or LP9000 can only write at around $15 \mathrm{MB} / \mathrm{s}$. Suspect the problem is related to the low write rates described in the previous two items. 


\title{
Appendix C: Individual Card performance (reference)
}

\author{
(all values in $\mathrm{MB} / \mathrm{s}$ )
}

IBM gigabit Ethernet NIC Performance (MB/s)

\begin{tabular}{|c|c|c|c|}
\multicolumn{1}{c}{ Architecture } & read & write & read/write \\
\hline IBM winterhawk2 & 105 & $100+$ & 160 \\
\hline IBM nighthawk2 * & 102 & 104 & 160 \\
\hline IBM M80 & 109 & 105 & 170 \\
\hline
\end{tabular}

* if you put multiple gige NIC's in a nighthawk RIO drawer, read rate for two NICs in a RIO (different buses) degrades to $82 \mathrm{MB} / \mathrm{s}$. Write rate degrades to $88 \mathrm{MB} / \mathrm{s}$ and aggregate degrades to $116 \mathrm{MB} / \mathrm{s}$.

Emulex LP8000 HBA Performance (MB/s)

\begin{tabular}{|c|c|c|c|}
\multicolumn{1}{c}{ Architecture } & read & write & read/write \\
\hline IBM winterhawk2 & 98 & 98 & 160 \\
\hline IBM nighthawk2* & $?$ & $?$ & $?$ \\
\hline IBM M80 & 98 & $77^{* *}$ & $112^{* *}$ \\
\hline
\end{tabular}

* if you put multiple HBA's in a nighthawk RIO drawer, data rates in a RIO drawer are limited by the RIO connection .... 250MB/s theoretical

** I suspect the problem with data rates is the emulex adapter NOT the M80!

This work was performed under the auspices of the U.S. Department of Energy by the University of California, Lawrence Livermore National Laboratory under Contract No. W-7405-Eng-48. 